AperTO - Archivio Istituzionale Open Access dell'Università di Torino

\title{
Pseudo-differential operators and existence of Gabor frames
}

\section{This is the author's manuscript}

Original Citation:

Availability:

This version is available http://hdl.handle.net/2318/1703985

since 2022-03-01T15:48:19Z

Published version:

DOI:10.1007/s11868-019-00279-1

Terms of use:

Open Access

Anyone can freely access the full text of works made available as "Open Access". Works made available under a Creative Commons license can be used according to the terms and conditions of said license. Use of all other works requires consent of the right holder (author or publisher) if not exempted from copyright protection by the applicable law. 


\title{
PSEUDO-DIFFERENTIAL OPERATORS AND EXISTENCE OF GABOR FRAMES
}

\author{
PAOLO BOGGIATTO AND GIANLUCA GARELLO
}

\begin{abstract}
We study from a pseudo-differential point of view the frame operator associated with a Gabor system. In particular we show how an application of the classical boundedness theorem of Calderón -Vaillancourt yields sufficient conditions for a Gabor system to form a frame in $L^{2}\left(\mathbb{R}^{d}\right)$.
\end{abstract}

\section{INTRODUCTION}

The central problem of time-frequency analysis is the extraction of information about the frequency content of a signal in dependence on time. In this context a signal is a complex function, or distribution, $f(t)$ of the time variable $t$, which for generality is usually supposed to be in $\mathbb{R}^{d}$. To this aim, Gabor frames, also known as Weyl-Heisenberg frames, have proved to be a particularly useful tool. Their theory has grown in the last decades to a vast subject of research in applied harmonic analysis, with connections to various aspects of abstract harmonic analysis.

At the core of Gabor frames is the idea of representing signals as expansions in terms of translations and modulations of a fixed analysing window function. More precisely suppose $g \in L^{2}\left(\mathbb{R}^{d}\right)$ is a non identically zero function, then for $\alpha, \beta \in \mathbb{R}_{+}$, its translations and modulations

$$
g_{h, k}(t)=e^{2 \pi i \beta k \cdot t} g(t-\alpha h), \quad h, k \in \mathbb{Z}^{d},
$$

are called time-frequency shifts of $g$ of parameter $\alpha, \beta$. The Gabor system

$$
\mathcal{G}(g, \alpha, \beta)=\left\{g_{h, k}\right\}_{h, k \in \mathbb{Z}^{d}}
$$

is said a Gabor frame in $L^{2}\left(\mathbb{R}^{d}\right)$ if there exist $A, B>0$ such that

$$
A\|f\|_{2}^{2} \leq \sum_{h, k}\left|\left(f, g_{h, k}\right)_{L^{2}}\right|^{2} \leq B\|f\|_{2}^{2},
$$

for every $f \in L^{2}\left(\mathbb{R}^{d}\right)$. In this case a classical result asserts the existence of frames $\left\{\widetilde{g}_{h, k}\right\}_{h, k \in \mathbb{Z}}$, called dual frames of $\left\{g_{h, k}\right\}_{h, k \in \mathbb{Z}}$, such that the following reconstruction formula holds:

$$
f=\sum_{h, k}\left(f, \widetilde{g}_{h, k}\right) g_{h, k},
$$

for all $f \in L^{2}\left(\mathbb{R}^{d}\right)$, with unconditional convergence in $L^{2}\left(\mathbb{R}^{d}\right)$.

The literature about Gabor frames theory is so vast that we do not attempt to give an exhausting references list, but we just indicate for example the monographs $[5],[8],[15],[20]$, and the references therein. A problem of great interest is to find conditions on the window $g(t)$ and on the parameters $\alpha, \beta$ in order that $\mathcal{G}(g, \alpha, \beta)$ is a Gabor frame. In the most part of the literature the conditions are only sufficient or necessary, characterizations are known only for few cases, see for instance [17] and [18] for some recent results of Gröchenig and Stöckler about totally positive

2000 Mathematics Subject Classification. Primary 42C15; Secondary 42C40, 47G30, 35S05.

Key words and phrases. Gabor frames, pseudo-differential operators, Calderón -Vaillancourt Theorem. 
functions. The general leading idea is that, for suitable fixed window $g$, if $\alpha$ and $\beta$ are "sufficiently small" then the lattice $\alpha \mathbb{Z}^{d} \times \beta \mathbb{Z}^{d}$ is refined enough to yield a frame. See [16], [19] for general surveys and, among others, [2], [4], [7], [9], [24], [25], [27], [28], [29], [30], [35] for specific contributions.

For any Gabor system $\mathcal{G}(g, \alpha, \beta)$ a Gabor operator may be formally defined by

$$
S f=\sum_{h, k}\left(f, g_{h, k}\right) g_{h, k}, \quad f \in \mathcal{S}\left(\mathbb{R}^{d}\right) .
$$

It is well known that the Gabor system $(0.2)$ is a frame in $L^{2}$ if and only if the related Gabor operator is invertible in $L^{2}$. In particular the invertibility of the Gabor operator may be proved by estimating its closeness to the identity. See for example [11] where such estimation is obtained with the aid of the spreading function, in the case of more general lattices $\Lambda=M \mathbb{Z}^{2 d}$, generated by a $2^{2 d} \times 2^{2 d}$ non singular matrix.

The results we present in this paper go exactly in this direction, but the technique used is not quite typical of time-frequency analysis. The main idea is to estimate the closeness of the Gabor operator to the identity by means of the classical Calderón -Vaillancourt Theorem for $L^{2}$-boundedness of pseudo-differential operators. We work with lattices $\Lambda=M \mathbb{Z}^{2 d}$, where $M$ is a diagonal matrix, therefore slightly generalizing the case $\alpha \mathbb{Z}^{d} \times \beta \mathbb{Z}^{d}$.

Namely our Gabor systems $\mathcal{G}(g, a, b)$ are defined, for $g$ measurable function, $a=\left(a_{1}, \ldots, a_{d}\right), b=\left(b_{1}, \ldots b_{d}\right)$ in $\mathbb{R}_{+}^{d}$, by

$$
\mathcal{G}(g, a, b)=\left\{g_{h, k}(t)=e^{2 \pi i b k \cdot t} g(t-a h)\right\}_{h, k \in \mathbb{Z}^{d}},
$$

where

$$
a h=\left(\begin{array}{ccc}
a_{1} & \ldots & 0 \\
\vdots & \ddots & \vdots \\
0 & \ldots & a_{d}
\end{array}\right)\left(\begin{array}{c}
h_{1} \\
\vdots \\
h_{d}
\end{array}\right) ; b k=\left(\begin{array}{ccc}
b_{1} & \ldots & 0 \\
\vdots & \ddots & \vdots \\
0 & \ldots & b_{d}
\end{array}\right)\left(\begin{array}{c}
k_{1} \\
\vdots \\
k_{d}
\end{array}\right)
$$

The aim is to find sufficient conditions on the vectors $a, b$ and the windows $g$ such that the Gabor operator (0.5) is a well-defined, bounded and invertible operator on $L^{2}\left(\mathbb{R}^{d}\right)$.

From a different perspective we remark that frames theory, and in general timefrequency analysis, has already revealed deep and interesting connections in a number of topics essentially related to pseudo-differential operators which act, or admit symbols in modulation or Wiener amalgam spaces, see for reference [1], [3], [6], [21], [26], [32], [33], [34].

The paper is organized as follows. In Section 1 we state the main results and in Section 2 we fix the notations and give the necessary definitions and tools.

In Section 3 we introduce a suitable class of pseudo-differential operators with periodic symbol. By means of Calderón -Vaillancourt Theorem we prove their boundedness and invertibility in $L^{2}\left(\mathbb{R}^{d}\right)$.

Incidentally we remark that we have chosen the Kohn-Nirenberg quantization in order to apply a convenient version of the Calderón -Vaillancourt Theorem. In principle however other quantizations can also be used.

In Section 4 we write $S$ as a pseudo-differential operator in the class described above and we prove that, for sufficiently small $a, b$, we have $\|I d-c S\|_{L^{2}}<1$, which, by the von Neumann series, implies the invertibility of the operator $S$. This invertibility is equivalent to the fact that $\left\{g_{h, k}\right\}$ is a frame and furnishes furthermore the frame bounds. The conditions on $a, b$ and $c$ depend only on the regularity and the decay at infinity of the window $g$.

We remark that the sufficient conditions on the density of the frame lattice, as well as the frames bounds that we determine in this paper are by far not optimal. 
The interest of the method relies instead on the fact that our results (Thm. 1.1, Cor. 1.2 ) are valid for a rather general class of windows in dimension $d$, whereas most of the more sharp results are obtained in one dimension and refer to specific windows. Moreover Theorem 3.7 about boundedness and invertibility of pseudo-differential operators with periodized symbols is interesting by itself in the framework of the pseudo-differential calculus.

We have chosen to remain in the $L^{2}$ setting since this is the natural context for the Calderón -Vaillancourt theorem and our methods are new even in this context. A reformulation in more general contexts, e.g. in the framework of Banach Gelfand triple setting, see [10], could be possible. In Section 5 we end the paper using the pseudo-differential form for the Gabor operator (0.5) to obtain a characterization for dual frames of $\left\{g_{h, k}\right\}$. Here we also point out the connections between our functional setting and the Feichtinger algebra $M^{1}$ (Lemma 5.1). To this regard see also [12] for results in the more general context of weak duality.

\section{Statement of the main Result}

We give here the essential tools for understanding the main result. More notations and definitions will be detailed in the next section.

For any $a, b \in \mathbb{R}_{+}^{d}$ and $x, \xi \in \mathbb{R}^{d}$ we define:

- $\langle x\rangle=\sqrt{1+|x|^{2}}$;

- $x \cdot \xi=\sum_{j=1}^{d} x_{j} \xi_{j}$;

- $a x=\left(a_{1} x_{1}, \ldots, a_{d} x_{d}\right)$;

- $\Pi a_{j} b_{j}=\Pi_{j=1}^{d} a_{j} b_{j}$;

- $T_{d}=\left\{\left(\alpha_{1}, \ldots, \alpha_{d}\right) \in \mathbb{Z}_{+}^{d} ;\right.$ such that $\alpha_{j}=0$ or 1$\}$.

For $f: \mathbb{R}^{d} \mapsto \mathbb{C}$ measurable function and $\varepsilon>0$ we say that

$$
\begin{aligned}
& f \in L_{\varepsilon}^{\infty}=L_{\varepsilon}^{\infty}\left(\mathbb{R}^{d}\right) \quad \text { if } \quad\|f\|_{L_{\varepsilon}^{\infty}}:=\left\|f(\cdot)\langle\cdot\rangle^{d+\varepsilon}\right\|_{L^{\infty}}<\infty, \\
& f \in \hat{L}_{\varepsilon}^{\infty} \text { if } \hat{f} \in L_{\varepsilon}^{\infty},
\end{aligned}
$$

where $\hat{f}$ is the Fourier transform of $f$. We write moreover $f \in \hat{C}^{d}$ if $\hat{f}$ belongs to the set of $d$ times differentiable functions.

The main result (Theorem 1.1) will make use of the following constants, depending only on the dimension $d$ and the positive parameter $\varepsilon$ :

$$
\begin{array}{ll}
N_{d}=3^{5 d}(2 \pi)^{\frac{5 d}{2}}(d+1)^{2 d}, & N_{d, \varepsilon}=(2(d+1))^{2 \varepsilon}\left(\int\langle x\rangle^{-d-\varepsilon} d x\right)^{2} \\
M_{d}=3^{5 d+1} 4^{d}(d+1)^{4 d}(2 \pi)^{\frac{5 d+2}{2}}, & M_{d, \varepsilon}=(2(d+1))^{4 \varepsilon}\left(\int\langle x\rangle^{-d-\varepsilon} d x\right)^{2} .
\end{array}
$$

Theorem 1.1. Consider $g \in C^{d+1} \cap \hat{C}^{d+1}$, such that, for any $\alpha, \beta \in T_{d}, j=$ $1, \ldots, d, x^{\alpha} \partial_{x}^{\beta} g, x^{\alpha} \partial_{x_{j}} \partial_{x}^{\beta} g, x_{j} x^{\alpha} \partial_{x}^{\beta} g$ belong to $L_{\varepsilon}^{\infty}\left(\mathbb{R}^{d}\right) \cap \hat{L}_{\varepsilon}^{\infty}\left(\mathbb{R}^{d}\right)$, for some $\varepsilon>0$. Assume that $a, b \in(0,1]^{d}$ satisfy the condition

$$
\sum_{j=0}^{d}\left(a_{j}+b_{j}\right)<\frac{\|g\|_{L^{2}}^{2}}{M_{d} M_{d, \varepsilon} \mathcal{K}_{g}}
$$

with $\mathcal{K}_{g}$ suitable positive constant depending on the norm of $g$ in $L_{\varepsilon}^{\infty}, \hat{L}_{\varepsilon}^{\infty}$. Then the Gabor system $\mathcal{G}(g, a, b)$ is a Gabor Frame in $L^{2}\left(\mathbb{R}^{d}\right)$. 
Moreover the frame bounds $A, B$ in (0.3) are

$$
\begin{aligned}
& A=\frac{\|g\|_{L^{2}}^{2}-M_{d} M_{d, \varepsilon} \mathcal{K}_{g} \sum_{j=1}^{d}\left(a_{j}+b_{j}\right)}{\Pi a_{j} b_{j}} \\
& B=N_{d} N_{d, \varepsilon} \max _{\alpha, \beta \in T_{d}}\left\{\left\|x^{\alpha} \partial_{x}^{\beta} g\right\|_{L_{\varepsilon}^{\infty}}\left\|\xi^{\beta} \partial_{\xi}^{\alpha} \hat{g}\right\|_{L_{\varepsilon}^{\infty}}\right\} .
\end{aligned}
$$

Precisely the constant $\mathcal{K}_{g}$ is:

$$
\mathcal{K}_{g}=\max _{\substack{\alpha, \beta \in T_{d} \\
j=1, \ldots, d}}\left\{\begin{array}{l}
\left\|x^{\alpha} \partial_{x}^{\beta} g\right\|_{L_{\varepsilon}^{\infty}}\left\|\xi^{\beta} \partial_{\xi}^{\alpha} \hat{g}\right\|_{L_{\varepsilon}^{\infty} ;} \\
\left\|x^{\alpha} \partial_{x}^{\beta} g\right\|_{L_{\varepsilon}^{\infty}}\left\|\xi_{j} \xi^{\beta} \partial_{\xi}^{\alpha} \hat{g}\right\|_{L_{\varepsilon}^{\infty}} ;\left\|x^{\alpha} \partial_{x_{j}} \partial_{x}^{\beta} g\right\|_{L^{\infty}}\left\|\xi^{\beta} \partial_{\xi}^{\alpha} \hat{g}\right\|_{L_{\varepsilon}^{\infty}} ; \\
\left\|x_{j} x^{\alpha} \partial_{x}^{\beta} g\right\|_{L_{\varepsilon}^{\infty}}\left\|\xi^{\beta} \partial_{\xi}^{\alpha} \hat{g}\right\|_{L_{\varepsilon}^{\infty}} ;\left\|x^{\alpha} \partial_{x}^{\beta} g\right\|_{L_{\varepsilon}^{\infty}}\left\|\xi^{\beta} \partial_{\xi_{j}} \partial_{\xi}^{\alpha} \hat{g}\right\|_{L_{\varepsilon}^{\infty}}
\end{array}\right\}
$$

The proof is obtained combining properties of frame operators in Hilbert spaces and a result of invertibility of pseudo-differential operators with periodic symbols, obtained by means of a careful application of the Calderón -Vaillancourt Theorem. The details are given in the next Section 4, after the preparation material of Sections 2 and 3.

Corollary 1.2. Consider $g \in C^{2 d+2}$, such that for any $\alpha \in T_{d},|\beta| \leq 2 d+1$, $j=1, \ldots, d, x^{\alpha} \partial_{x}^{\beta} g, x^{\alpha} \partial_{x_{j}} \partial_{x}^{\beta} g, x_{j} x^{\alpha} \partial_{x}^{\beta} g$ belong to $L_{\varepsilon}^{\infty}\left(\mathbb{R}^{d}\right)$. Then, with the same assumption on $a, b \in[0,1)^{d}$ and the same involved constants, all the results in Theorem 1.1 are true.

Example 1.3. Despite the apparently complicated hypotheses, we notice that the previous results apply to very simple types of window function, which do not belong to the Schwartz space. For example in one dimension the functions

$$
g(x)=\frac{1}{p+q x^{4 k}}, \quad p, q>0, \quad k=1,2,3, \ldots
$$

satisfy the hypothesis of Theorem 1.1.

Windows (essentially) of this type are of considerable interest; actually results of Janssen [23] furnish a complete characterization of the Gabor sampling set for windows of the type $\left(1+a x^{2}\right)^{-1}, a>0$.

Let us notice that in the particular case $a_{j}=\alpha>0, b_{j}=\beta>0, j=1, . ., d$, we have the bounded inclusion $L_{\varepsilon}^{\infty}\left(\mathbb{R}^{d}\right) \hookrightarrow W\left(\mathbb{R}^{d}\right)$, where $W\left(\mathbb{R}^{d}\right)$ is the Wiener function space (see [15], Def. 6.1.1). In this case our results overlap with results of Walnut [35], where however conditions on the sampling parameters $\alpha, \beta$, and as consequence the frame bounds $A, B$, are given in different way.

Finally, due to the inequality $\|\cdot\|_{2} \leq C\|\cdot\|_{L_{\varepsilon}^{\infty}}, C=\left(\int\langle x\rangle^{-2(d+\varepsilon)} d x\right)^{1 / 2}$, we can notice that the sufficient condition (1.5) implies the estimate $\sum_{j=0}^{\infty}\left(a_{j}+b_{j}\right)<$ $C / M_{d} M_{d, \varepsilon}$, independently of $g$. We conclude that, as noticed in the Introduction, the bound in the right-hand side of (1.5) is far from being optimal.

\section{Notations AND BACKGRound}

Given $f(x), u(x, t)$ in the Schwartz classes of rapidly decreasing functions $\mathcal{S}\left(\mathbb{R}^{d}\right)$ and $\mathcal{S}\left(\mathbb{R}^{2 d}\right)$ respectively, we define the Fourier transforms $\mathcal{F} f(\xi)=\hat{f}(\xi)=$ $\int e^{-2 \pi i x \omega} f(x) d x, \mathcal{F}_{2} u(x, \xi)=\int e^{-2 \pi i t \omega} u(x, t) d t$, togheter with the inverse transforms: $\mathcal{F}^{-1} f(\xi)=\check{f}(\xi)=\int e^{2 \pi i x \omega} f(x) d x, \mathcal{F}_{2}^{-1} u(x, \xi)=\int e^{2 \pi i t \omega} u(x, t) d t$. In the same way we denote their extension to $\mathcal{S}^{\prime}\left(\mathbb{R}^{d}\right)$ and $\mathcal{S}^{\prime}\left(\mathbb{R}^{2 d}\right)$.

Let us recall the following basic properties which will be useful in the sequel.

i) If $x^{\alpha} f(x) \in L^{1}\left(\mathbb{R}^{d}\right),|\alpha| \leq N$, then $\hat{f} \in C^{N}\left(\mathbb{R}^{d}\right)$ and

$$
\mathcal{F}\left((-2 \pi i x)^{\alpha} f\right)(\xi)=\partial^{\alpha}(\mathcal{F} f)(\xi), \quad|\alpha| \leq N .
$$


ii) If $f \in C^{N}\left(\mathbb{R}^{d}\right)$ and $\partial_{x}^{\alpha} f \in L^{1}\left(\mathbb{R}^{d}\right),|\alpha| \leq N$, then

$$
\mathcal{F}\left(\partial^{\alpha} f\right)(\xi)=(2 \pi i \xi)^{\alpha} \mathcal{F} f(\xi) .
$$

Time frequency shifts. For $y, \omega \in \mathbb{R}^{d}$ we define the operators:

$$
\begin{array}{lll}
T_{y} f(t)=f(t-y), & \ddot{T}_{y} u(x, \xi)=u(x, \xi-y) & \text { (translation); } \\
M_{\omega} f(t)=e^{2 \pi i \omega \cdot t} f(t), & \ddot{M}_{\omega} u(x, \xi)=e^{2 \pi i \omega \cdot \xi} u(x, \xi) & \text { (modulation). }
\end{array}
$$

The next properties follow:

$$
\begin{array}{ll}
\mathcal{F}\left(T_{y} f\right)=M_{-y} \mathcal{F} f, & \mathcal{F}^{-1}\left(T_{y} f\right)=M_{y} \mathcal{F}^{-1} f, \\
\mathcal{F}\left(M_{\omega} f\right)=T_{\omega} \mathcal{F} f, & \mathcal{F}^{-1}\left(M_{\omega} f\right)=T_{-\omega} \mathcal{F}^{-1} f, \\
\mathcal{F}\left(M_{\omega} T_{y} f\right)=T_{\omega} M_{-y} \mathcal{F} f ; & \mathcal{F}^{-1}\left(T_{y} M_{\omega} f\right)=M_{-y} T_{\omega} \mathcal{F}^{-1} f .
\end{array}
$$

Function spaces. Together with the definition of $L_{\varepsilon}^{\infty}$ and $\hat{L}_{\varepsilon}^{\infty}$, given in (1.1), $(1.2)$, we define $L_{\varepsilon, \varepsilon}^{\infty}\left(\mathbb{R}^{2 d}\right)$ as the set of the measurable functions $p(x, \xi)$ on $\mathbb{R}_{x}^{d} \times \mathbb{R}_{\xi}^{d}$ such that

$$
\|p\|_{L_{\varepsilon, \varepsilon}^{\infty}}:=\left\|p(x, \xi)\langle x\rangle^{d+\varepsilon}\langle\xi\rangle^{d+\varepsilon}\right\|_{L^{\infty}\left(\mathbb{R}^{2 d}\right)}<\infty .
$$

Notice that for any $\varepsilon>0, L_{\varepsilon}^{\infty}\left(\mathbb{R}^{d}\right) \subset L^{1}\left(\mathbb{R}^{d}\right)$ and $L_{\varepsilon, \varepsilon}^{\infty}\left(\mathbb{R}^{2 d}\right) \subset L^{1}\left(\mathbb{R}^{2 d}\right)$. Since for some $C>0$ : $\langle x\rangle^{d+\varepsilon / 2}\langle\xi\rangle^{d+\varepsilon / 2} \leq C\langle(x, \xi)\rangle^{2 d+\varepsilon}$, we obtain for any $\varepsilon>0$

$$
L_{\varepsilon}^{\infty}\left(\mathbb{R}^{2 d}\right) \subset L_{\frac{\varepsilon}{2}, \frac{\varepsilon}{2}}^{\infty}\left(\mathbb{R}^{2 d}\right) .
$$

We say moreover that the function $p(x, \xi)$ is $(a, b)$-periodic, $a, b \in \mathbb{R}^{d}$, if for any $m, n \in \mathbb{Z}^{d}$ we have $p(x, \xi)=p(x+m a, \xi+n b)$

Invertibility in Banach algebras. We will make use of the properties of the von Neumann series in Banach algebras of operators in the following version

Proposition 2.1. Consider $x \in \mathcal{A}$, where $(\mathcal{A},\|\cdot\|)$ is a Banach algebra on the field of complex numbers, with multiplicative identity e. If there exist $c \in \mathbb{C} \backslash\{0\}$ such $\|e-c x\|<1$ then $x$ is invertible in $\mathcal{A}$ and

$$
x^{-1}=c \sum_{n=0}^{\infty}(e-c x)^{n} .
$$

Frames in Hilbert Spaces. A sequence $\left\{x_{n}\right\}_{n \in \mathbb{N}}$ in a separable Hilbert space $H$ is a frame if there exist $A, B>0$ such that $A\|x\|^{2} \leq \sum_{n}\left|\left(x, x_{n}\right)\right|^{2} \leq B\|x\|^{2}$ for $x \in H$.

Notice now that for any sequence $\left\{x_{n}\right\}_{n \in \mathbb{N}} \subset H$ we can formally define the operator

$$
S: x \rightarrow S x=\sum_{n \in \mathbb{N}}\left(x, x_{n}\right) x_{n}, \quad x \in H .
$$

The main result in Theorem 1.1 is based on the fact that $\left\{x_{n}\right\}_{n \in \mathbb{N}} \subset H$ is a frame if and only if $S$ is a bijection of $H$ in itself.

Although this result is well-known it is not easy to find it explicitly mentioned and proved in the literature, therefore we give next the details.

Lemma 2.2 (Heil [20]). $\left\{x_{n}\right\}_{n \in \mathbb{N}}$ is a frame for $H$ if and only if there exist $A, B>0$ such that $A I \leq S \leq B I$.

Proposition 2.3. $\left\{x_{n}\right\}_{n \in \mathbb{N}}$ is a frame for $H$ if and only of $S: H \rightarrow H$ is a bijection. Furthermore in this case $S$ is bi-continuous. 
Proof. See [20] for the proof that if $\left\{x_{n}\right\}_{n \in \mathbb{N}}$ is a frame then $S$ is a bijection.

On the converse, suppose $S: H \rightarrow H$ is a bijection. Notice that for every $N \in \mathbb{N}$ the operators $S_{N}: x \in H \rightarrow S_{N} x=\sum_{n=1}^{N}\left(x, x_{n}\right) x_{n} \in H$ are bounded, namely $\left\|S_{N} x\right\| \leq \sum_{n=1}^{N}\left|\left(x, x_{n}\right)\right|\left\|x_{n}\right\| \leq\|x\| \sum_{n=1}^{N}\left\|x_{n}\right\|^{2}$.

Clearly $\lim _{N \rightarrow \infty} S_{N} x=S x$ for every $x \in H$. Then from the Banach-Steinhaus theorem follows that $S$ is bounded. From the Banach inverse operator theorem also $S^{-1}$ is bounded.

For every $x \in H,(S x, x)=\sum_{n \in \mathbb{N}}\left|\left(x, x_{n}\right)\right|^{2} \geq 0$, therefore $S \geq 0$.

As $S \geq 0$ and is invertible, the same holds for $S^{-1}$, namely for $y=S x$ we have $\left(S^{-1} y, y\right)=(x, S x) \geq 0$.

From the boundedness of $S$ we have $(S x, x) \leq\|S x\|\|x\| \leq\|S\|\|x\|^{2}=\|S\|(x, x)$, i.e. $S \leq\|S\| I$.

Analogously, as $S^{-1}$ continuous, $\left(S^{-1} y, y\right) \leq\left\|S^{-1} y\right\|\|y\| \leq\|S\|\|y\|^{2}=\|S\|(y, y)$, i.e. $S^{-1} \leq\left\|S^{-1}\right\| I$.

Multiplying this inequality by $S$ we get $\frac{1}{\left\|S^{-1}\right\|} I \leq S$. It follows $\frac{1}{\left\|S^{-1}\right\|} I \leq S \leq\|S\| I$, and this means that $\left\{x_{n}\right\}_{n \in \mathbb{N}}$ is a frame by the previous Lemma.

Multiple expansions. We prove here a technical result about multiple expansions.

For fixed $j \leq d$, consider a set of indices $J \subset\{1, \ldots, d\}$, containing exactly $j$ elements and set:

$$
\begin{aligned}
& K_{J}=:\left\{h \in \mathbb{Z}^{d} ; \begin{array}{ll}
h_{i} \neq 0, & i \in J \\
h_{i}=0, & i \notin J
\end{array}\right\} \\
& \mathbb{Z}_{0}^{m}=:\left\{h \in \mathbb{Z}^{m} ; h_{j} \neq 0, \text { for any } j=1, \ldots, m\right\} \\
& \mathbb{Z}_{0,+}^{m}=:\left\{h \in \mathbb{Z}^{m} ; h_{j}>0, \text { for any } j=1, \ldots, m\right\}
\end{aligned}
$$

Lemma 2.4. For any $f: \mathbb{R}^{d} \mapsto \mathbb{C}, a \in \mathbb{R}_{+}^{d}, N>0$, we have:

$$
\begin{aligned}
& \sum_{h \in \mathbb{Z}^{d}} f(h)=\sum_{j=0}^{d} \sum_{|J|=j} \sum_{h \in K_{J}} f(h) \\
& \sum_{h \in \mathbb{Z}_{0}^{m}}\langle a h\rangle^{-N}=2^{m} \sum_{h \in \mathbb{Z}_{0,+}^{m}}\langle a h\rangle^{-N}, \quad m=1, \ldots, d
\end{aligned}
$$$$
\sum_{h \in \mathbb{Z}_{0,+}^{m}}\langle a h\rangle^{-N} \leq \frac{1}{\prod_{i} a_{i}} \int_{[0, \infty)^{m}}\langle x\rangle^{-N} d x, \quad m=1, \ldots, d
$$

$$
\sum_{a \in \mathbb{Z}^{d}}\langle a h\rangle^{-N} \leq 1+M_{a, d} \int_{[0, \infty)^{d}}\langle x\rangle^{-N} d x, \quad M_{a, d}=\sum_{j=1}^{d} 2^{j} \sum_{|J|=j} \frac{1}{\prod_{i \in J} a_{i}}
$$

Proof. Notice that $|\{J: J \subset\{1, \ldots d\}\}|=\left(\begin{array}{c}d \\ j\end{array}\right)$. We can prove (2.15) by induction on the dimension $d$. Namely:

For $d=1, \sum_{h \in \mathbb{Z}} f(h)=\sum_{j=0}^{1} \sum_{n=1}^{\left(\begin{array}{l}1 \\ j\end{array}\right)} \sum_{h \in K_{J}} f(h)=f(0)+\sum_{h \neq 0} f(h)$ is verified. 
Assume now that (2.15) holds for $d-1$, then:

$$
\begin{aligned}
& \sum_{h \in \mathbb{Z}^{d}} f(h)=\sum_{\tilde{h} \in \mathbb{Z}^{d-1}} f(\tilde{h}, 0)+\sum_{h_{d} \neq 0} \sum_{\tilde{h} \in \mathbb{Z}^{d-1}} f\left(\tilde{h}, h_{d}\right)= \\
& =\sum_{j=0}^{d-1} \sum_{\substack{J \subseteq\{1, \ldots, d-1\} \\
|J|=j}} \sum_{\tilde{h} \in K_{J}} f(\tilde{h}, 0)+\sum_{h_{d} \neq 0} \sum_{j=0}^{d-1} \sum_{\substack{J=\{1, \ldots, d-1\} \\
|J|=j}} \sum_{\tilde{h} \in K_{J}} f\left(\tilde{h}, h_{d}\right)= \\
& =\sum_{j=0}^{d} \sum_{\substack{J \subseteq\{1, \ldots, d\} \\
|\bar{J}|=j, d \notin J}} \sum_{h \in K_{J}} f(h)+\sum_{j=1}^{d} \sum_{\substack{J=\{1, \ldots, d\} \\
|J|=j, d \in J}} \sum_{h \in K_{J}} f(h)= \\
& =\sum_{j=0}^{d} \sum_{\substack{J \subseteq\{1, \ldots, d\} \\
|J|=j}} \sum_{h \in K_{J}} f(h)
\end{aligned}
$$

(2.16) directly follows by induction on the index $m$, since it is trivial for $m=1$ and

$$
\sum_{h \in \mathbb{Z}_{0}^{m}}\langle a h\rangle^{-N}=\sum_{h_{m} \neq 0} 2^{m-1} \sum_{h \in \mathbb{Z}_{0,+}^{m-1}}\left\langle a h, a_{m} h_{m}\right\rangle=2^{m} \sum_{h \in \mathbb{Z}_{0,+}^{m}}\langle a h\rangle^{-N}
$$

Thanks to a classic result about multiple expansions, see e.g. [14], we obtain that the expansion in (2.17) converges if and only if the multiple integral $\int_{[0,+\infty)^{m}}\langle a x\rangle^{-N} d x$ is convergent, moreover

$$
\sum_{h \in \mathbb{Z}_{0,+}^{m}}\langle a h\rangle^{-N} \leq \int_{[0,+\infty)^{m}}\langle a x\rangle^{-N} \leq \sum_{h \in \mathbb{Z}_{+}^{m}}\langle a x\rangle^{-N}
$$

Using both (2.16) and (2.17), we obtain

$$
\begin{aligned}
\sum_{h \in \mathbb{Z}^{d}}\langle a h\rangle^{-N} & =\sum_{j=0}^{d} \sum_{|J|=j} \sum_{h \in K_{J}}\langle a h\rangle^{-N} \\
& \leq 1+\sum_{j=1}^{d} 2^{j} \sum_{|J|=j} \frac{1}{\prod_{i \in J}^{a_{i}}} \int_{\tilde{x} \in[0, \infty)^{j}}\langle\tilde{x}\rangle^{-N} d \tilde{x} \\
& \leq 1+\int_{x \in[0, \infty)^{d}}\langle x\rangle^{-N} d x \sum_{j=1}^{d} 2^{j} \sum_{|J|=j} \frac{1}{\prod_{i \in J}^{a_{i}}} .
\end{aligned}
$$

Setting now $M_{a, d}=\sum_{j=1}^{d} 2^{j} \sum_{|J|=j} \frac{1}{\prod_{i \in J} a i}$ the proof is concluded.

Remark 2.5. If $a_{j} \leq 1, j=1, \ldots, d$, we can notice that $M_{a, d} \leq \frac{1}{\Pi a_{i}} \sum_{j=1}^{d} 2^{j}\left(\begin{array}{l}d \\ j\end{array}\right)=$ $\frac{1}{\Pi a_{j}}\left(3^{d}-1\right)$, then $1+M_{a, d} \int_{[0,+\infty)^{d}}\langle x\rangle^{-d-\varepsilon} d x \leq \frac{1}{\Pi a_{j}}\left(\frac{3}{2}\right)^{d} \int\langle x\rangle^{-\bar{d}-\varepsilon} d x$. We can then conclude that

$$
\sum_{h \in \mathbb{Z}^{d}}\langle a h\rangle^{-d-\varepsilon} \leq \frac{1}{\Pi a_{j}}\left(\frac{3}{2}\right)^{d} \int\langle x\rangle^{-d-\varepsilon} d x, \quad \text { when } \quad a \in(0,1]^{d}, \varepsilon>0 .
$$

\section{Pseudo-differential Operators}

Recall that, for $a(x, \xi) \in \mathcal{S}^{\prime}\left(\mathbb{R}^{2 d}\right)$, the Kohn-Nirenberg quantization of $a(x, \xi)$ is the pseudo-differential operator which defines the bounded map

$$
\varphi \in \mathcal{S}\left(\mathbb{R}^{d}\right) \longrightarrow a(x, D) \varphi=\mathcal{F}_{2}^{-1}(a(x, \xi) \hat{\varphi}(\xi)) \in \mathcal{S}^{\prime}\left(\mathbb{R}^{d}\right) .
$$


We notice that

$$
\begin{aligned}
a(x, D) \varphi(x) & =\iint e^{2 \pi i \xi \cdot(x-t)} a(x, \xi) \varphi(t) d t d \xi \\
& =\int \varphi(t) \int e^{-2 \pi i(t-x) \cdot \xi} a(x, \xi) d \xi d t \\
& =\left\langle\left(\mathcal{F}_{2}(x, \cdot-x), \varphi\right\rangle=\left\langle\ddot{T}_{x}\left(\mathcal{F}_{2} a\right)(x, \cdot), \varphi\right\rangle,\right.
\end{aligned}
$$

and the following kernel theorem holds.

Proposition 3.1. For any $a \in \mathcal{S}^{\prime}\left(\mathbb{R}^{2 d}\right)$ the pseudo-differential operator $a(x, D)$ can be expressed as kernel operator, precisely for any $\varphi \in \mathcal{S}$ we have:

$$
\begin{gathered}
a(x, D) \varphi=\langle K(x, \cdot), \varphi\rangle, \quad \text { where } \\
K(x, t)=\ddot{T}_{x}\left(\mathcal{F}_{2} a\right)(x, t) \quad \text { or equivalently } a(x, \xi)=\ddot{M}_{-x}\left(\mathcal{F}_{2}^{-1} K\right)(x, \xi) .
\end{gathered}
$$

Concerning the $L^{2}$ boundedness of pseudo-differential operators let us recall the Calderón -Vaillancourt Theorem in the version of Hwang [22, Theorem 2].

Theorem 3.2. Let $a: \mathbb{R}^{d} \times \mathbb{R}^{d} \mapsto \mathbb{C}$ be a continuous function whose derivatives $\partial_{x}^{\alpha} \partial_{x}^{\beta}$ a satisfy the following condition:

$$
\begin{aligned}
& \text { there is a constant } C>0 \text { such that }\left\|\partial_{x}^{\alpha} \partial_{\xi}^{\beta} a\right\|_{L^{\infty}\left(\mathbb{R}^{2 d}\right)} \leq C, \\
& \text { where } \alpha=\left(\alpha_{1}, \ldots, \alpha_{d}\right), \beta=\left(\beta_{1}, \ldots, \beta_{d}\right) \in \mathbb{Z}_{+}^{d} \\
& \text { with } \alpha_{j}=0 \text { or } 1, \beta_{j}=0 \text { or } 1 .
\end{aligned}
$$

Then $a(x, D)$ is continuous from $L^{2}\left(\mathbb{R}^{d}\right)$ to $L^{2}\left(\mathbb{R}^{d}\right)$, with norm estimate $\|a(x, D)\| \leq$ $C_{d}\|a\| \|$, where $C_{d}$ is a constant depending only on $d$ and $\|\mid a\|$ is the smallest $C$ such that (3.5) holds.

Remark 3.3. Arguing carefully on the proof of Theorem 2 in [22], assuming that the conditions (3.5) are satisfied, we can write for any $u, v \in C_{0}^{\infty}\left(\mathbb{R}^{d}\right)$,

$$
\langle a(x, D) u, v\rangle=\sum_{\alpha \in T_{d}} \sum_{\beta \leq \alpha}(-1)^{|\alpha|}\left(\frac{1}{2 \pi}\right)^{2 d} \int \partial_{x}^{\alpha-\beta} b(x, \xi) g_{\beta}(x, \xi) h(x, \xi) d x d \xi
$$

where $\left\|g_{\beta}\right\|_{L^{2}} \leq(2 \pi)^{d / 2} \pi^{d / 2}\|u\|_{L^{2}} ;\|h\|_{L^{2}}=(2 \pi)^{d} \pi^{d / 2}\|v\|_{L^{2}} ;\left\|\partial_{x}^{\alpha-\beta} b\right\|_{L^{\infty}} \leq 2^{d}\||| a\|$. Thus

$$
|\langle a(x, D) u, v\rangle| \leq(2 \pi)^{d / 2} K_{d}\|a\| u\left\|_{L^{2}}\right\| v \|_{L^{2}}, \quad K_{d}=\sum_{\alpha \in T_{d}} \sum_{\beta \leq \alpha} 1 .
$$

It may be easily proved by induction on the dimension $d$, that $K_{d}=3^{d}$. Namely $K_{1}=\sum_{\alpha_{1}=0}^{1} \sum_{\beta_{1}=0}^{\alpha_{1}} 1=3$; assuming now that $K_{d}=3^{d}$ we have $K_{d+1}=\sum_{\alpha \in T_{d+1}} \sum_{\beta \leq \alpha} 1=$ $\sum_{\alpha_{d+1}=0}^{1} \sum_{\beta_{d+1}=0}^{\alpha_{d+1}} K_{d}=3^{d+1}$

We can conclude that the constant in Theorem 3.2 is

$$
C_{d}=3^{d}(2 \pi)^{d / 2} \text {. }
$$

Lemma 3.4. Consider the following periodization of $p(x, \xi) \in L_{\varepsilon, \varepsilon}^{\infty}\left(\mathbb{R}^{2 d}\right)$ :

$$
\sum_{h, k \in \mathbb{Z}^{d}} p(x-a h ; \xi-b k) .
$$

Then for any $a, b \in(0,1]^{d}$ we have:

i) the sum in $(3.9)$ is convergent in $L^{\infty}\left(\mathbb{R}^{2 d}\right)$, and it satisfies the estimate:

$$
\left.\| \sum_{h, k \in \mathbb{Z}^{d}} p(x-a h, \xi-b k)\right)\left\|_{L^{\infty}\left(\mathbb{R}^{2 d}\right)} \leq v_{\varepsilon, d}^{2} \frac{1}{\Pi a_{j} b_{j}}\right\| p \|_{L_{\varepsilon, \varepsilon}^{\infty}}
$$


with

$$
v_{\varepsilon, d}=3^{d} 2^{\varepsilon}(d+1)^{d+\varepsilon} \int\langle y\rangle^{-d-\varepsilon} d y
$$

ii) If moreover $p(x, \xi)$ is a continuous function, then the sum in (3.9) is a continuous function of $x, \xi \in \mathbb{R}^{2 d}$.

Remark that $i$ ) and $i i$ ) are still valid for general $a, b \in \mathbb{R}_{+}^{d}$, but the constants $v_{\varepsilon, d}$ also depends on $\langle a\rangle^{d+\varepsilon},\langle b\rangle^{d+\varepsilon}$ and on $M_{a, d}$ and $M_{b, d}$ defined in (2.18).

Proof. Since the expression in (3.9) is $(a, b)$ periodic, for any $(x, \xi) \in \mathbb{R}^{2 d}$ we can find $(\bar{x}, \bar{\xi})$ in the thorus $\mathbb{T}_{a, b}^{2 d}:=\Pi_{j=1}^{d}\left(\left[0, a_{j}\right] \times\left[0, b_{j}\right]\right)$, such that $\sum_{h, k \in \mathbb{Z}^{d}} p(x-a h, \xi-$ $b k)=\sum_{h, k \in \mathbb{Z}^{d}} p(\bar{x}-a h, \bar{\xi}-b k)$. Considering moreover that $p \in L_{\varepsilon, \varepsilon}^{\infty}, a, b \in(0,1]^{d}$, using Peetre's inequality and estimate $(2.22)$ we have for almost any $(x, \xi) \in \mathbb{R}^{2 d}$ :

$$
\begin{aligned}
& \sum_{h, k \in \mathbb{R}^{2 d}}|p(x-a h, \xi-b k)|=\sum_{h, k \in \mathbb{Z}^{d}}|p(\bar{x}-a h, \bar{\xi}-b k)| \leq \\
\leq & \sum_{h, k \in \mathbb{Z}^{d}} \operatorname{ess} \sup _{x, \xi \in \mathbb{T}_{a, b}^{2 d}}\left[|p(\bar{x}-a h, \bar{\xi}-b k)|\langle\bar{x}-a h\rangle^{d+\varepsilon}\langle\bar{\xi}-b k\rangle^{d+\varepsilon} \times\right. \\
& \left.\times\langle\bar{x}-a h\rangle^{-d-\varepsilon}\langle\bar{\xi}-b k\rangle^{-d-\varepsilon}\right] \leq \\
\leq & 2^{2(d+\varepsilon)}\langle\bar{x}\rangle^{d+\varepsilon}\langle\bar{\xi}\rangle^{d+\varepsilon}\|p\|_{L_{\varepsilon, \varepsilon}^{\infty}} \sum_{h, k \in \mathbb{Z}^{d}}\langle a h\rangle^{-d-\varepsilon}\langle b k\rangle^{-d-\varepsilon} \leq \\
\leq & 2^{2(d+\varepsilon)} 3^{2 d}\langle a\rangle^{d+\varepsilon}\langle b\rangle^{d+\varepsilon} \frac{1}{\Pi a_{j} b_{j}} \frac{1}{2^{2 d}}\left(\int\langle y\rangle^{-d-\varepsilon} d y\right)^{2}\|p\|_{L_{\varepsilon, \varepsilon}^{\infty}} \leq \\
\leq & 3^{2 d} 2^{2 \varepsilon}(d+1)^{2(d+\varepsilon)}\left(\int\langle y\rangle^{-d-\varepsilon} d y\right)^{2} \frac{1}{\Pi a_{j} b_{j}}\|p\|_{L_{\varepsilon, \varepsilon}^{\infty} .}
\end{aligned}
$$

When $p(x, \xi)$ is continuous, the $L^{\infty}$ convergence implies the uniform convergence, thus the sum is continuous.

In the following we consider, for $p \in L_{\varepsilon, \varepsilon}^{\infty}\left(\mathbb{R}^{2 d}\right)$, the symbol

$$
\varsigma(x, \xi)=\sum_{h, k \in \mathbb{Z}^{d}} p(x-a h, \xi-b k), \quad a, b \in[0,1)^{d} .
$$

Since the expansion in (3.9) is convergent in $\mathcal{S}^{\prime}\left(\mathbb{R}^{2 d}\right)$, thanks to (3.10) we have, for any $\alpha, \beta \in \mathbb{Z}_{+}^{d}$ :

$$
\left\|\partial_{\xi}^{\alpha} \partial_{x}^{\beta} \varsigma(x, \xi)\right\|_{L^{\infty}}=\left\|\sum_{h, k \in \mathbb{Z}^{d}} \partial_{\xi}^{\alpha} \partial_{x}^{\beta} p(x-a h, \xi-b k)\right\|_{L^{\infty}} \leq \frac{v_{\varepsilon, d}}{\Pi_{a_{j} b_{j}}}\left\|\partial_{\xi}^{\alpha} \partial_{x}^{\beta} p\right\|_{L_{\varepsilon, \varepsilon}^{\infty}} .
$$

Applying now the Calderón -Vaillancourt Theorem, we can state the following:

Proposition 3.5. Consider a continuous function $p(x, \xi)$ such that, for any $\alpha, \beta \in$ $T_{d}$, we have $\partial_{\xi}^{\alpha} \partial_{x}^{\beta} p \in L_{\varepsilon, \varepsilon}^{\infty}\left(\mathbb{R}^{2 d}\right)$. Then the operator $\varsigma(x, D)$ is bounded on $L^{2}\left(\mathbb{R}^{d}\right)$ with norm estimate:

$$
\|\varsigma(x, D)\|_{\mathcal{L}\left(L^{2}\right)} \leq \frac{3^{d}(2 \pi)^{d / 2} v_{\varepsilon, d}^{2}}{\Pi a_{j} b_{j}} \max _{\alpha, \beta \in T_{d}}\left\{\left\|\partial_{\xi}^{\alpha} \partial_{x}^{\beta} p\right\|_{L_{\varepsilon, \varepsilon}^{\infty}}\right\} .
$$

Lemma 3.6. Consider $p \in C^{N+1}\left(\mathbb{R}^{2 d}\right)$ and assume that $\partial_{\xi}^{\alpha} \partial_{x}^{\beta} p \in L_{\varepsilon, \varepsilon}^{\infty}$, for $|\alpha+\beta| \leq$ $N+1$. Then for any $a, b \in(0,1]^{d}$ we have 
i) If $N=0$

$$
\begin{aligned}
& \left\|\int p(y, \eta) d y d \eta-\Pi a_{j} b_{j} \sum_{h, h \in \mathbb{Z}^{d}} p\left(x-a h, \xi-b_{k}\right)\right\|_{L^{\infty}\left(\mathbb{R}^{2 d}\right)} \leq \\
\leq & \kappa_{\varepsilon, d}^{2} \sum_{j=1}^{d} a_{j}\left\|\partial_{x_{j}} p\right\|_{L_{\varepsilon, \varepsilon}^{\infty}}+b_{j}\left\|\partial_{\xi_{j}} p\right\|_{L_{\varepsilon, \varepsilon}^{\infty},}
\end{aligned}
$$

where

$$
\kappa_{\varepsilon, d}=3^{d} 2^{d} 2^{2 \varepsilon}(d+1)^{2(d+\varepsilon)} \int\langle x\rangle^{-d-\varepsilon} d x
$$

ii) If $N \geq 1$ and $0<|\alpha+\beta| \leq N$

$$
\begin{array}{r} 
\\
\quad\left\|\Pi a_{j} b_{j} \sum_{h, k \in \mathbb{Z}^{d}} \partial_{\xi}^{\alpha} \partial_{x}^{\beta} p(x-a h, \xi-b k)\right\|_{L^{\infty}\left(\mathbb{R}^{2 d}\right)} \leq \\
\leq \kappa_{\varepsilon, d}^{2} \sum_{j=1}^{d} a_{j}\left\|\partial_{x_{k}} \partial_{\xi}^{\alpha} \partial_{x}^{\beta} p\right\|_{L_{\varepsilon, \varepsilon}^{\infty}}+b_{j}\left\|\partial_{\xi_{k}} \partial_{\xi}^{\alpha} \partial_{x}^{\beta} p\right\|_{L_{\varepsilon, \varepsilon}^{\infty},},
\end{array}
$$

with $\kappa_{\varepsilon, d}$ as before.

Proof. Since $\int p(y, \eta) d y d \eta$ is a constant and $\sum_{h, k \in \mathbb{Z}^{d}} p(x-a h, \xi-b k)$ is $(a, b)$ periodic, we can reduce the norm in the left-hand side of (3.16) to the essential supremum on the Thorus $\mathbb{T}_{a, b}^{2 d}=\Pi_{j=1}^{d}\left[0, a_{j}\right] \times\left[0, b_{j}\right]$.

Consider now the intervals $I_{h, k}=\Pi_{j=1}^{d}\left[a_{j} h_{j} ; a_{j}\left(h_{j}+1\right)\right) \times\left[b_{j} k_{j} ; b_{j}\left(k_{j}+1\right)\right)$. Notice that $\bigcup_{h, k \in \mathbb{Z}^{d}} I_{h, k}=\mathbb{R}^{2 d}, I_{h, k} \cap I_{m, n}=\emptyset$ if $(h, k) \neq(m, n)$. By setting $\tilde{p}(y, \eta)=p(-y,-\eta)$ and observing that the measure of any interval $I_{h, k}$ is $\left|I_{h, k}\right|=$ $\Pi a_{j} b_{j}$, by means of a suitable change of variable we obtain, for any $(x, \xi) \in \mathbb{T}_{a, b}^{2 d}$ :

$$
\begin{aligned}
& \left|\int p(y, \eta) d y d \eta-\Pi a_{j} b_{j} \sum_{h, h \in \mathbb{Z}^{d}} p(x-a h, \xi-b k)\right|= \\
= & \left|\int T_{x, \xi} \tilde{p}(y, \eta) d y d \eta-\sum_{h, h \in \mathbb{Z}^{d}}\right| I_{h, k}\left|T_{x, \xi} \tilde{p}(a h, b k)\right|= \\
= & \left|\sum_{h, h \in \mathbb{Z}^{d}} \int_{I_{h, k}} T_{x, \xi} \tilde{p}(y, \eta) d y d \eta-\sum_{h, h \in \mathbb{Z}^{d}} \int_{I_{h, k}} T_{x, \xi} \tilde{p}(a h, b k) d y d \eta\right| \leq \\
\leq & \sum_{h, k \in \mathbb{Z}^{d}} \int_{I_{h, k}}\left|T_{x, \xi} \tilde{p}(y, \eta)-T_{x, \xi} \tilde{p}(a h, b k)\right| d y d \eta \leq \\
\leq & \sum_{h, k \in \mathbb{Z}^{d}}\left|I_{h, k}\right| \sup _{(y, \eta) \in I_{h, k}}\left|T_{x, \xi} \tilde{p}(y, \eta)-T_{x, \xi} \tilde{p}(a h, b k)\right| \leq \\
\leq & \prod_{a_{j} b_{j}} \sum_{h, k \in \mathbb{Z}^{d}} \sup _{(y, \eta) \in I_{h, k}}\left|T_{x, \xi} \tilde{p}(y, \eta)-T_{x, \xi} \tilde{p}(a h, b k)\right| .
\end{aligned}
$$

Assuming that $p \in C^{1}\left(\mathbb{R}^{2 d}\right)$ and $\partial_{\xi}^{\alpha} \partial_{x}^{\beta} p \in L_{\varepsilon, \varepsilon}^{\infty}$ when $|\alpha+\beta| \leq 1$, the Taylor expansion with integral remainder and the Peetre's inequality give the following 
estimate for any $(y, \eta) \in I_{h, k}$ and $(h, k) \in \mathbb{Z}^{d}$ :

$$
\begin{aligned}
& \left.\mid T_{x, \xi} \tilde{p}(y, \eta)-T_{x, \xi} \tilde{p}(a h, b k)\right) \mid= \\
= & \sum_{j=1}^{d}\left|y_{j}-a_{j} h_{j}\right| \int_{0}^{1}\left|\partial_{y_{j}} T_{x, \xi} \tilde{p}(a h+t(y-a h) ; b k+t(\eta-b k))\right| d t+ \\
+ & \left|\eta_{j}-b_{j} k_{j}\right| \int_{0}^{1}\left|\partial_{\eta_{j}} T_{x, \xi} \tilde{p}(a h+t(y-a h) ; b k+t(\eta-b k))\right| d t \leq \\
\leq & \sum_{j=1}^{d} a_{j} \int_{0}^{1}\left|\partial_{y_{j}} p(x-a h-t(y-a h) ; \xi-b k-t(\eta-b k))\right| d t+ \\
+ & b_{j} \int_{0}^{1}\left|\partial_{\eta_{j}} p(x-a h-t(y-a h) ; \xi-b k-t(\eta-b k))\right| d t \leq \\
\leq & \int_{0}^{1}\langle x-a h-t(y-a h)\rangle^{-d-\varepsilon}\langle\xi-b k-t(\eta-b k)\rangle^{-d-\varepsilon} d t \times \\
& \times \sum_{j=1}^{d} a_{j}\left\|\partial_{y_{j}} p\right\|_{L_{\varepsilon, \varepsilon}^{\infty}}+b_{j}\left\|\partial_{\xi_{j}} p\right\|_{L_{\varepsilon, \varepsilon}^{\infty}} \leq \\
\leq & 4^{d+\varepsilon}\langle x-a h\rangle^{-d-\varepsilon}\langle\xi-b k\rangle^{-d-\varepsilon} \int_{0}^{1}\langle t(y-a h)\rangle^{d+\varepsilon}\langle t(\eta-b k)\rangle^{d+\varepsilon} d t \times \\
& \times \sum_{j=1} a_{j}\left\|\partial_{x_{j}} p\right\|_{L_{\varepsilon, \varepsilon}^{\infty}}+b_{j}\left\|\partial_{\xi_{j}} p\right\|_{L_{\varepsilon, \varepsilon}^{\infty}}^{\infty} \leq \\
\leq & (16\langle x\rangle\langle\xi\rangle\langle a\rangle\langle b\rangle)^{d+\varepsilon} \sum_{j=1}^{d} a_{j}\left\|\partial_{x_{j}} p\right\|_{L_{\varepsilon, \varepsilon}^{\infty}}\left\|b_{j} \partial_{\xi_{j}} p\right\|_{L_{\varepsilon, \varepsilon}^{\infty}}\langle a h\rangle^{-d-\varepsilon\langle b k\rangle^{-d-\varepsilon} .}
\end{aligned}
$$

Thus recalling that $a, b \in(0,1]^{d}$ and using (2.22), we obtain

$$
\begin{aligned}
& \left\|\int p(y, \eta) d y d \eta-\Pi a_{j} b_{j} \sum_{h, h \in \mathbb{Z}^{d}} p\left(x-a h, \xi-b_{k}\right)\right\|_{L^{\infty}\left(\mathbb{R}^{2 d}\right)} \\
\leq & 2^{4(d+\varepsilon)}(d+1)^{4(d+\varepsilon)} \Pi a_{j} b_{j} \sum_{j=1}^{d} a_{j}\left\|\partial_{x_{j}} p\right\|_{L_{\varepsilon, \varepsilon}^{\infty}}+b_{j}\left\|\partial_{\xi_{j}} p\right\|_{L_{\varepsilon, \varepsilon}^{\infty}} \sum_{h, k \in \mathbb{Z}^{d}}\langle a h\rangle^{-d-\varepsilon}\langle b k\rangle^{-d-\varepsilon} \\
\leq & 2^{2 d} 2^{4 \varepsilon} 3^{2 d}(d+1)^{4(d+\varepsilon)}\left(\int\langle x\rangle^{-d-\varepsilon} d x\right)^{2} \Pi a_{j} b_{j} \sum_{j=1}^{d} a_{j}\left\|\partial_{x_{j}} p\right\|_{L_{\varepsilon, \varepsilon}^{\infty}}+b_{j}\left\|\partial_{\xi_{j}} p\right\|_{L_{\varepsilon, \varepsilon}^{\infty}} .
\end{aligned}
$$

By setting $\kappa_{\varepsilon, d}=3^{d} 2^{d} 2^{\varepsilon}(d+1)^{2(d+\varepsilon)} \int\langle x\rangle^{-d-\varepsilon} d x$, the proof of $\left.i\right)$ is concluded.

In order to prove $i i)$ notice that there exists $j=1, \ldots, d$ such that at least one among $\alpha_{j}$ or $\beta_{j}$ is different from 0 (assume $a_{j} \neq 0$ ). Moreover $\partial_{\xi}^{\alpha_{1} \ldots\left(\alpha_{j}-1\right) \ldots \alpha_{d}} \partial_{x}^{\beta} p \in$ $L_{\varepsilon, \varepsilon}^{\infty}$, that is it vanishes at infinity. Since $\partial_{\xi}^{\alpha} \partial_{x}^{\beta} p \in L^{1}\left(\mathbb{R}^{2 d}\right)$, applying the Fubini's Theorem we obtain

$$
\begin{aligned}
& \int \partial_{\xi}^{\alpha} \partial_{x}^{\beta} p(x, \xi) d \xi d x= \\
& \int_{\mathbb{R}_{x}^{d}} d x \int_{\mathbb{R}_{\xi_{i \neq j}^{d-1}}^{d-1}} d \xi_{i \neq j} \int_{\mathbb{R}_{\xi_{j}}} \partial_{\xi_{j}} \partial^{\alpha_{1} \ldots\left(\alpha_{j}-1\right) \ldots \alpha_{d}} \partial_{x}^{\beta} p(x, \xi) d \xi_{j}=0 .
\end{aligned}
$$

The proof then follows observing that $\partial_{\xi}^{\alpha} \partial_{x}^{\beta} p$ satisfies the assumptions in $i$ ).

Theorem 3.7. Consider $p(x, \xi) \in C^{2 d+1}\left(\mathbb{R}^{2 d}\right)$ such that, $\int p(x, \xi) d x d \xi \neq 0$ and for any $\alpha, \beta \in T_{d}, j=1, \ldots, d, \partial_{\xi}^{\alpha} \partial_{x}^{\beta} p, \partial_{x_{j}} \partial_{\xi}^{\alpha} \partial_{x}^{\beta} p$ and $\partial_{\xi_{j}} \partial_{\xi}^{\alpha} \partial_{x}^{\beta} p$ belong to $L_{\varepsilon, \varepsilon}^{\infty}\left(\mathbb{R}^{2 d}\right)$. Set

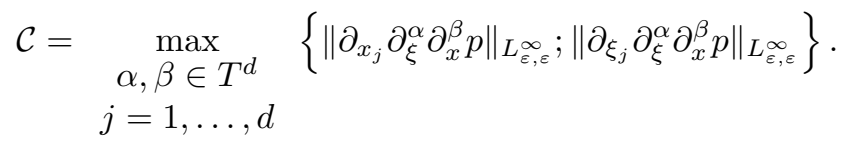

Then, for any $a, b \in(0,1]^{d}$ :

i) The operator $I-\frac{\Pi a_{j} b_{j}}{\int p(x, \xi) d x d \xi} \varsigma(x, D)$ is continuous from $L^{2}\left(\mathbb{R}^{d}\right)$ to itself and its operator norm satisfies:

$$
\left\|I-\frac{\Pi a_{j} b_{j}}{\int p(x, \xi) d x d \xi} \varsigma(x, D)\right\|_{\mathcal{L}\left(L^{2}\right)} \leq \frac{3^{d}(2 \pi)^{d / 2} \mathcal{C} \kappa_{\varepsilon, d}^{2}}{\left|\int p(x, \xi) d x d \xi\right|} \sum_{j=1}^{d}\left(a_{j}+b_{j}\right),
$$

where $\kappa_{\varepsilon, d}$ defined in $(3.17)$; 
ii) If moreover

$$
\sum_{j=1}^{d} a_{j}+b_{j}<\frac{\left|\int p(x, \xi) d x d \xi\right|}{3^{d}(2 \pi)^{d / 2} \mathcal{C} \kappa_{\varepsilon, d}^{2}}
$$

then the operator $\varsigma(x, D)$ is invertible in $\mathcal{L}\left(L^{2}\left(\mathbb{R}^{d}\right)\right)$ and we have:

$$
\begin{gathered}
\varsigma(x, D)^{-1}=\frac{\Pi a_{j} b_{j}}{\int p(x, \xi) d x d \xi} \sum_{n=0}^{\infty}\left(I-\frac{\Pi a_{j} b_{j}}{\int p(x, \xi) d x d \xi} \varsigma(x, D)\right)^{n} ; \\
\left\|\varsigma(x, D)^{-1}\right\|_{\mathcal{L}\left(L^{2}\right)} \leq \frac{\Pi a_{j} b_{j}}{\left|\int p(x, \xi) d x d \xi\right|-3^{3 d}(2 \pi)^{d / 2} \mathcal{C} \kappa_{\varepsilon, d}^{2} \sum_{j=1}^{d}\left(a_{j}+b_{j}\right)}
\end{gathered}
$$

Proof. $i$ ) is a straightforward application of Lemma 3.6, Theorem 3.2, jointly with Remark 3.3.

Working now in the Banach algebra $\left.\mathcal{L}\left(L^{2}\right), i i\right)$ easily follows from (3.22), (3.23), by setting $x=I-\frac{\Pi a_{j} b_{j}}{\int p(x, \xi) d x d \xi} \varsigma(x, D)$ in Proposition 2.1. The estimate (3.25) directly follows from the well known identity $\sum_{n=0}^{\infty} t^{n}=\frac{1}{1-t}$ when $|t|<1$.

\section{GABOr OpERATORS}

For $a, b \in \mathbb{R}_{+}^{d}, g$ measurable function, consider the Gabor systems $\mathcal{G}(g, a, b)$ and $G(\gamma, a, b)$.

Assume $g \in L_{\varepsilon}^{\infty}, \gamma \in \hat{L}_{\varepsilon}^{\infty}$ and $a, b \in \mathbb{R}_{+}^{d}$, then for any $\phi, \varphi \in \mathcal{S}\left(\mathbb{R}^{d}\right)$ :

$$
\begin{aligned}
& \left|\left\langle g_{h, k}, \varphi\right\rangle\left\langle\gamma_{h, k}, \phi\right\rangle\right|=\left|\left\langle g_{h, k}, \varphi\right\rangle\left\langle\widehat{\gamma_{h, k}}, \hat{\phi}\right\rangle\right|=\left|\left\langle M_{b k} T_{a h} g, \varphi\right\rangle\left\langle T_{b k} M_{-a h} \hat{\gamma}, \hat{\phi}\right\rangle\right| \leq \\
\leq & \left\|T_{a h} g\right\|_{L^{\infty}}\|\varphi\|_{L^{1}}\left\|T_{b k} \hat{\gamma}\right\|_{L^{\infty}}\|\phi\|_{L^{1}} .
\end{aligned}
$$

Directly from Lemma 3.4 we obtain that the expansion $\sum_{h, k \in \mathbb{Z}^{d}}\left(\varphi, g_{h, k}\right) \gamma_{h, k}$ is convergent in $\mathcal{S}^{\prime}\left(\mathbb{R}^{d}\right)$, for any $\varphi \in \mathcal{S}\left(\mathbb{R}^{d}\right)$.

For any $g \in L_{\varepsilon}^{\infty}, \gamma \in \hat{L}_{\varepsilon}^{\infty}$ we can then define the operator from $\mathcal{S}\left(\mathbb{R}^{d}\right)$ to $\mathcal{S}^{\prime}\left(\mathbb{R}^{d}\right)$ :

$$
S_{g, \gamma} \varphi=\sum_{h, k \in \mathbb{Z}^{d}}\left(\varphi, g_{h, k}\right) \gamma_{h, k} ; \quad \varphi \in \mathcal{S}\left(\mathbb{R}^{d}\right)
$$

which is said (generalized) Gabor operator. For $g \in L_{\varepsilon}^{\infty} \cap \hat{L}_{\varepsilon}^{\infty}$, we write $S_{g}=S_{g, g}$.

The explicit form of the kernel of $S_{g, \gamma}$ is given, for any $\varphi \in \mathcal{S}\left(\mathbb{R}^{d}\right)$, by

$$
S_{g, \gamma} \varphi(x)=\langle K(x, \cdot), \varphi\rangle, \quad \text { with } \quad K(x, t)=\sum_{h, k \in \mathbb{Z}^{d}} \bar{g}_{h k}(t) \gamma_{h k}(x),
$$

where the sum converges in $\mathcal{S}^{\prime}\left(\mathbb{R}^{2 d}\right)$.

The next result shows how a Gabor operator may be expressed as pseudodifferential operator with suitable periodic symbol.

Theorem 4.1. For $\gamma \in L_{\varepsilon}^{\infty}\left(\mathbb{R}^{d}\right), g \in \hat{L}_{\varepsilon}^{\infty}\left(\mathbb{R}^{d}\right)$ we obtain $S_{g, \gamma}=\sigma(x, D)$, where:

$$
\sigma(x, \xi)=\sum_{h, k \in \mathbb{Z}^{d}} e^{-2 \pi i(x-a h) \cdot(\xi-b k)} \gamma(x-a h) \overline{\hat{g}}(\xi-b k),
$$

with convergence in $L^{\infty}\left(\mathbb{R}^{2 d}\right)$. Moreover, when $a, b \in(0,1]^{d}$, its norm satisfies the following estimate:

$$
\|\sigma(x, \xi)\|_{\infty} \leq \frac{v_{\varepsilon, d}^{2}}{\Pi a_{j} b_{j}}\|\hat{g}\|_{L_{\varepsilon}^{\infty}}\|\gamma\|_{L_{\varepsilon}^{\infty}}
$$

with $v_{\varepsilon, d}$ defined in (3.11). 
Proof. Using (4.3) and (3.4) we have:

$$
\begin{aligned}
& \sigma(x, \xi)=\ddot{M}_{-x}\left(\mathcal{F}_{2}^{-1} K\right)(x, \xi)=\ddot{M}_{-x} \mathcal{F}_{2}^{-1}\left(\sum_{h, k \in \mathbb{Z}^{d}} \gamma_{h k}(x) \bar{g}_{h k}(t)\right)(\xi)= \\
& \ddot{M}_{-x} \sum_{h, k \in \mathbb{Z}^{d}} \gamma_{h k}(x)\left(\mathcal{F}_{2}{ }^{-1} \bar{g}_{h k}\right)(\xi)=\ddot{M}_{-x} \sum_{h, k \in \mathbb{Z}^{d}} \gamma_{h k} \mathcal{F}_{2}^{-1}\left(M_{-b k} T_{a h} \bar{g}\right)(\xi)= \\
& \ddot{M}_{-x} \sum_{h, k \in \mathbb{Z}^{d}} \gamma_{h k}(x) T_{b k} M_{a h} \overline{\bar{g}}(\xi)= \\
& e^{-2 \pi i x \cdot \xi} \sum_{h, k \in \mathbb{Z}^{d}} e^{2 \pi i k b \cdot x} \gamma(x-a h) T_{b k} M_{a h} \overline{\hat{g}}(\xi)= \\
& e^{-2 \pi i x \cdot \xi} \sum_{h, k \in \mathbb{Z}^{d}} e^{2 \pi i b k \cdot x} \gamma(x-a h) e^{2 \pi i a h \cdot(\xi-b k)} \overline{\hat{g}}(\xi-b k)= \\
& \sum_{h, k \in \mathbb{Z}^{d}} e^{-2 \pi i(x-a h) \cdot(\xi-b k)} \gamma(x-a h) \overline{\hat{g}}(\xi-b k) .
\end{aligned}
$$

The convergence of the expansion, estimate (4.5) and the continuity of $\sigma(x, \xi)$, directly follow from the conditions on the functions $g, \gamma$ and Lemma 3.4.

Lemma 4.2. Consider $u, v \in \mathcal{S}^{\prime}\left(\mathbb{R}^{d}\right)$, set $a(x, \xi)=e^{-2 \pi i x \cdot \xi} u(x) v(\xi)$, then for any $\alpha, \beta \in \mathbb{Z}_{+}^{d}$ we have

(4.6) $\partial_{\xi}^{\alpha} \partial_{x}^{\beta} a(x, \xi)=\sum_{\lambda \leq \alpha} \sum_{\mu \leq \lambda} C_{\lambda, \nu, \mu}^{\alpha, \beta}(-2 \pi i)^{|\lambda+\nu-\mu|} e^{-2 \pi i x \cdot \xi} U_{\lambda, \nu, \mu}(x) V_{\lambda, \nu, \mu}(\xi)$

$$
\nu \leq \beta \quad \mu \leq \nu
$$

(4.7) with $\quad U_{\lambda, \nu, \mu}^{\beta}(x)=x^{\lambda-\mu} \partial_{x}^{\beta-\nu} u(x), \quad V_{\lambda, \nu, \mu}^{\alpha}(\xi)=\xi^{\nu-\mu} \partial_{\xi}^{\alpha-\lambda} v(\xi)$

(4.8) and $C_{\lambda, \nu, \mu}^{\alpha, \beta}=\left(\begin{array}{l}\alpha \\ \lambda\end{array}\right)\left(\begin{array}{l}\beta \\ \nu\end{array}\right)\left(\begin{array}{l}\nu \\ \mu\end{array}\right)\left(\begin{array}{l}\lambda \\ \mu\end{array}\right) \mu !$.

If moreover $\alpha, \beta \in T_{d}, j=1, \ldots, d$ we obtain the estimate:

$$
\begin{gathered}
\left\|\partial_{\xi}^{\alpha} \partial_{x}^{\beta} a(x, \xi)\right\|_{L^{\infty}} \leq 3^{|\alpha+\beta|}(2 \pi)^{|\alpha+\beta|} \max _{\substack{\lambda, \mu \leq \alpha \\
\nu, \eta \leq \beta}}\left\{\left\|x^{\lambda} \partial_{x}^{\nu} u\right\|_{L^{\infty}}\left\|\xi^{\eta} \partial_{\xi}^{\mu} v\right\|_{L^{\infty}}\right\} . \\
\left\|\partial_{x_{j}} \partial_{\xi}^{\alpha} \partial_{x}^{\beta} a(x, \xi)\right\|_{L^{\infty}} \leq 3^{|\alpha+\beta|+1}(2 \pi)^{|\alpha+\beta|+1} \mathcal{M}_{\alpha}^{\beta}(u, v) \\
\left\|\partial_{\xi_{j}} \partial_{\xi}^{\alpha} \partial_{x}^{\beta} a(x, \xi)\right\|_{L^{\infty}} \leq 3^{|\alpha+\beta|+1}(2 \pi)^{|\alpha+\beta|+1} \mathcal{N}_{\alpha}^{\beta}(u, v)
\end{gathered}
$$

with

$$
\begin{gathered}
\mathcal{M}_{\alpha}^{\beta}(u, v)=\max _{\substack{\lambda, \mu \leq \alpha \\
\nu, \eta \leq \beta \\
j=1, \ldots, d}}\left\{\begin{array}{l}
\left\|x^{\lambda} \partial_{x}^{\nu} u\right\|_{L^{\infty}}\left\|\xi^{\eta} \partial_{\xi}^{\mu} v\right\|_{L^{\infty}}, \\
\left\|x^{\lambda} \partial_{x}^{\nu} u\right\|_{L^{\infty}}\left\|\xi_{j} \xi^{\nu} \partial_{\xi}^{\mu} v\right\|_{L^{\infty}}, \\
\left\|x^{\lambda} \partial_{x_{j}} \partial_{x}^{\nu} u\right\|_{L^{\infty}}\left\|\xi^{\eta} \partial_{\xi}^{\mu} v\right\|_{L^{\infty}}
\end{array}\right\}, \\
\mathcal{N}_{\alpha}^{\beta}(u, v)=\max _{\substack{\lambda, \mu \leq \alpha \\
\nu, \eta, \eta \\
j=1, \ldots, d}}\left\{\begin{array}{l}
\left\|x^{\lambda} \partial_{x}^{\nu} u\right\|_{L^{\infty}}\left\|\xi^{\eta} \partial_{\xi}^{\mu} v\right\|_{L^{\infty}}, \\
\left\|x_{j} x^{\lambda} \partial_{x}^{\nu} u\right\|_{L^{\infty}}\left\|\xi^{\nu} \partial_{\xi}^{\mu} v\right\|_{L^{\infty}}, \\
\left\|x^{\lambda} \partial_{x}^{\nu} u\right\|_{L^{\infty}}\left\|\xi^{\eta} \partial_{\xi_{j}} \partial_{\xi}^{\mu} v\right\|_{L^{\infty}}
\end{array}\right\} .
\end{gathered}
$$

Proof. By a straighforward application of the Leibnitz rule we have:

$$
\begin{aligned}
& \partial_{x}^{\beta} \partial_{\xi}^{\alpha} a(x, \xi)=\partial_{x}^{\beta} u(x) \sum_{\lambda \leq \alpha}\left(\begin{array}{l}
\alpha \\
\lambda
\end{array}\right) \partial_{\xi}^{\alpha}(-2 \pi i)^{|\lambda|} x^{\lambda} e^{-2 \pi i x \cdot \xi} \partial_{\xi}^{\alpha-\lambda} v(\xi)= \\
& =\sum_{\lambda \leq \alpha}\left(\begin{array}{l}
\alpha \\
\lambda
\end{array}\right)(-2 \pi i)^{|\lambda|} \partial^{\alpha-\lambda} v(\xi) \sum_{\nu \leq \beta}\left(\begin{array}{l}
\beta \\
\nu
\end{array}\right) \partial_{x}^{\nu}\left(x^{\lambda} e^{-2 \pi i x \cdot \xi}\right) \partial^{\beta-\nu} u(x)= \\
& =\sum_{\lambda \leq \alpha} \sum_{\mu \leq \lambda}\left(\begin{array}{l}
\alpha \\
\lambda
\end{array}\right)\left(\begin{array}{l}
\beta \\
\nu
\end{array}\right)\left(\begin{array}{l}
\nu \\
\mu
\end{array}\right)(-2 \pi i)^{|\lambda+\nu-\mu|} \frac{\lambda !}{(\lambda-\mu) !} \times \\
& \nu \leq \beta \mu \leq \nu
\end{aligned}
$$

$$
\times e^{-2 \pi i x \cdot \xi} x^{\lambda-\mu} \xi^{\nu-\mu} \partial^{\alpha-\lambda} v(\xi) \partial^{\beta-\nu} u(x) .
$$


By noticing that $\frac{\lambda !}{(\lambda-\mu) !}=\left(\begin{array}{c}\lambda \\ \mu\end{array}\right) \mu$ !, setting $U_{\lambda, \nu, \mu}^{\alpha}(x), V_{\lambda, \nu, \mu}^{\beta}(\xi)$ and $C_{\lambda, \nu, \mu}^{\alpha, \beta}$ as in (4.7), (4.8), we obtain (4.6). Estimate (4.9) directly follows by observing that $\mu$ ! $=1$, when $\alpha, \beta \in T^{d}$ and by means of a suitable application of Newton's binomial identity:

$$
(x+y)^{\alpha}=\sum_{\beta \leq \alpha}\left(\begin{array}{c}
\alpha \\
\beta
\end{array}\right) x^{\alpha-\beta} y^{\beta}, \quad x, y \in \mathbb{R}^{d}, \quad \alpha \in \mathbb{Z}_{+}^{d} .
$$

Setting $e_{j}=\left(0, \ldots, 0, \alpha_{j}=1,0, \ldots 0\right)$ we have:

$$
\partial_{x_{j}} U_{\lambda, \nu, \mu}(x)=\left(\lambda_{j}-\mu_{j}\right) x^{\lambda-\mu-e_{j}} \partial_{x}^{\beta-\nu} u(x)+x^{\lambda-\mu} \partial_{x}^{\beta-\nu+e_{j}} u(x),
$$

thus

$$
\partial_{x_{j}} \partial_{\xi}^{\alpha} \partial_{x}^{\beta} a(x, \xi)=\sum_{\substack{\lambda \leq \alpha \\ \nu \leq \beta}} \sum_{\substack{\mu \leq \lambda \\ \mu \leq \nu}} C_{\lambda, \nu, \mu}^{\alpha \cdot \beta}(2 \pi i)^{|\lambda-\mu-\nu|} e^{-2 \pi i x \cdot \xi} \triangle(x, \xi)
$$

with

$$
\begin{aligned}
\triangle(x, \xi)= & (2 \pi i) \xi_{j} \xi^{\nu-\mu} \partial_{\xi}^{\alpha-\lambda} v(\xi) x^{\lambda-\mu} \partial_{x}^{\beta-\nu} u(x)+ \\
& \left(\lambda_{j}-\mu_{j}\right) \xi^{\nu-\mu} \partial_{\xi}^{\alpha-\lambda} v(\xi) x^{\lambda-\mu-e_{j}} \partial_{x}^{\beta-\nu} u(x)+ \\
& \xi^{\nu-\mu} \partial_{\xi}^{\alpha-\lambda} v(\xi) x^{\lambda-\mu} \partial_{x_{j}} \partial_{x}^{\beta-\nu} u(x) .
\end{aligned}
$$

Assuming that $\alpha, \beta \in T^{d}$, observing that $\mu_{j} \leq \lambda_{j} \leq \alpha_{j} \leq 1$ and arguing as in proof of (4.9), we obtain (4.10). Simmetrically we can prove (4.11).

Thanks to Theorem 4.1 the Gabor operator $S_{g, \gamma}$ is a pseudo-differential operator with symbol obtained as periodization of $p(x, \xi)=e^{-2 \pi i x \xi} \gamma(x) \overline{\hat{g}}(\xi)$. Using then Theorem 3.2, Theorem 3.7, Lemma 4.2 and observing that $\int p(x, \xi) d x d \xi=(\gamma, g)$, we obtain the following invertibility result.

Theorem 4.3. Consider the functions $\gamma \in C^{d+1}, g \in \hat{C}^{d+1}$ such that, for any $\alpha, \beta \in T_{d}$ and $j=1, \ldots, d, x^{\alpha} \partial_{x}^{\beta} \gamma, x^{\alpha} \partial_{x_{j}} \partial_{x}^{\beta} \gamma, x_{j} x^{\alpha} \partial_{x}^{\beta} \gamma$ belong to $L_{\varepsilon}^{\infty}\left(\mathbb{R}^{d}\right)$ and $x^{\alpha} \partial_{x}^{\beta} g, x^{\alpha} \partial_{x_{j}} \partial_{x}^{\beta} g, x_{j} x^{\alpha} \partial_{x}^{\beta} g$ belong to $\hat{L}_{\varepsilon}^{\infty}\left(\mathbb{R}^{d}\right)$.

set:

$$
\mathcal{K}_{g, \gamma}=\max _{\substack{\alpha, \beta \in T_{d} \\
j=1, \ldots, d}}\left\{\begin{array}{lll}
\left\|x^{\alpha} \partial_{x}^{\beta} \gamma\right\|_{L_{\varepsilon}^{\infty}}\left\|\xi^{\beta} \partial_{\xi}^{\alpha} \hat{g}\right\|_{L_{\varepsilon}^{\infty}} & ; \quad\left\|x^{\alpha} \partial_{x}^{\beta} \gamma\right\|_{L_{\varepsilon}^{\infty}}\left\|\xi_{j} \xi^{\beta} \partial_{\xi}^{\alpha} \hat{g}\right\|_{L_{\varepsilon}^{\infty}} \\
\left\|x^{\alpha} \partial_{x_{j}} \partial_{x}^{\beta} \gamma\right\|_{L_{\varepsilon}^{\infty}\left\|\xi^{\beta} \partial_{\xi}^{\alpha} \hat{g}\right\|_{L_{\varepsilon}^{\infty}}} & ; \quad\left\|x_{j} x^{\alpha} \partial_{x}^{\beta} \gamma\right\|_{L_{\varepsilon}^{\infty}}\left\|\xi^{\beta} \partial_{\xi}^{\alpha} \hat{g}\right\|_{L_{\varepsilon}^{\infty} ;} ; \\
\left\|x^{\alpha} \partial_{x}^{\beta} \gamma\right\|_{L_{\varepsilon}^{\infty}}\left\|\xi^{\beta} \partial_{\xi_{j}} \partial_{\xi}^{\alpha} \hat{g}\right\|_{L_{\varepsilon}^{\infty}} &
\end{array}\right\}
$$

then for some positive constants $M_{d}, M_{d, \varepsilon}, N_{d}, N_{d, \varepsilon}$ we have

i) For any $a, b \in(0,1]^{d}$ the operator $S_{g, \gamma}$ is $L^{2}$ bounded and moreover

$$
\begin{gathered}
\left\|S_{g, \gamma}\right\|_{\mathcal{L}\left(L^{2}\right)} \leq N_{d} N_{d, \varepsilon} \max _{\alpha, \beta \in T_{d}}\left\{\left\|x^{\alpha} \partial_{x}^{\beta} g\right\|_{L_{\varepsilon}^{\infty}}\left\|\xi^{\beta} \partial_{\xi}^{\alpha} \hat{g}\right\|_{L_{\varepsilon}^{\infty}}\right\} \\
\left\|I-\frac{\Pi a_{j} b_{j}}{(\gamma, g)} S_{g, \gamma}\right\|_{\mathcal{L}\left(L^{2}\right)} \leq M_{d} M_{d, \varepsilon} \frac{\mathcal{K}_{g, \gamma}}{|(\gamma, g)|} \sum_{j=1}^{d}\left(a_{j}+b_{j}\right)
\end{gathered}
$$

ii) If moreover $\sum_{j=1}^{d}\left(a_{j}+b_{j}\right)<\frac{|(\gamma, g)|}{C_{d} C_{d, \varepsilon} \mathcal{K}_{g, \gamma}}$, then the operator $S_{g, \gamma}$ is invertible in $\mathcal{L}\left(L^{2}\left(\mathbb{R}^{d}\right)\right)$, more precisely

$$
S_{g, \gamma}^{-1}=\frac{\Pi a_{j} b_{j}}{|(\gamma, g)|} \sum_{n=0}^{\infty}\left(I-\frac{\Pi a_{j} b_{j}}{|(\gamma, g)|} S_{g, \gamma}\right)^{n},
$$

and

$$
\left\|S_{g, \gamma}^{-1}\right\|_{\mathcal{L}\left(L^{2}\right)} \leq \frac{\Pi a_{j} b_{j}}{|(\gamma, g)|-M_{d} M_{d, \varepsilon} \mathcal{K}_{g, \gamma} \sum_{j=1}^{d}\left(a_{j}+b_{j}\right)}
$$


The constants $M_{d}, M_{d, \varepsilon}, N_{d}, N_{d, \varepsilon}$ depend only on $d$ and $\varepsilon$, precisely:

$$
\begin{array}{ll}
N_{d}=3^{5 d}(2 \pi)^{\frac{5 d}{2}}(d+1)^{2 d}, & N_{d, \varepsilon}=(2(d+1))^{2 \varepsilon}\left(\int\langle x\rangle^{-d-\varepsilon} d x\right)^{2} ; \\
M_{d}=3^{5 d+1} 4^{d}(d+1)^{4 d}(2 \pi)^{\frac{5 d+2}{2}}, & M_{d, \varepsilon}=(2(d+1))^{4 \varepsilon}\left(\int\langle x\rangle^{-d-\varepsilon} d x\right)^{2} .
\end{array}
$$

Now applying Proposition 2.3 we obtain the main result of the paper: Theorem 1.1 .

\section{Proof of Corollary 1.2}

Proof. In order to prove Corollary 1.2, let us notice that in general $\xi^{\beta} \partial_{\xi}^{\alpha} \hat{g}(\xi) \in L_{\varepsilon}^{\infty}$ if $\left(\langle\xi\rangle^{d+1+|\beta|} \widehat{x^{\alpha} g}(\xi) \in L^{\infty}\right.$. Thanks to (2.1), (2.2), the last statement is verified if $x^{\alpha} g \in C^{d+1+|\beta|}$ and, for any $|\gamma| \leq d+1+|\beta|, \partial_{x}^{\gamma}\left(x^{\alpha} g(x)\right)=\sum_{\eta \leq \gamma} c_{\eta} x^{\alpha-\eta} \partial^{\gamma-\eta} g(x)$ belongs to $L_{\varepsilon}^{\infty} \subset L^{1}$. Remembering we need that $\xi^{\beta} \partial_{\xi}^{\alpha} \hat{g}, \xi_{j} \xi^{\beta} \partial_{\xi}^{\alpha} \hat{g}$ and $\xi^{\beta} \partial_{\xi_{j}} \partial_{\xi}^{\alpha} \hat{g}$ belong to $L_{\varepsilon}^{\infty}$, when $\alpha, \beta \in T_{d}$, with straightforward calculation we obtain the results in Corollary 1.2 with the assumptions: $g \in C^{2 d+2}, x^{\alpha} \partial^{\beta} g, x^{\alpha} \partial_{x_{j}} \partial_{x}^{\beta} g, x_{j} x^{\alpha} \partial_{x}^{\beta} g$ belong to $L_{\varepsilon}^{\infty}$, for any $\alpha \in T_{d},|\beta| \leq 2 d+1, j=1, \ldots, d$.

\section{A characterization of dual Gabor windows}

By means of the pseudo-differential calculus, we characterize in this section when two windows in the modulation space $g, \gamma \in M^{1}$ generate dual Gabor frames in terms of their $\tau$-Wigner transform (for the definition of $M^{1}$ and more general modulation spaces see for example [15], Ch. 11). As Lemma 5.1 will show, the condition $g, \gamma \in M^{1}$ is a generalization, for suitably large $\varepsilon$, of the condition $\gamma, g \in L_{\varepsilon}^{\infty}\left(\mathbb{R}^{d}\right) \cap \hat{L}_{\varepsilon}^{\infty}\left(\mathbb{R}^{d}\right)$ which was considered in the paragraphs before.

We start by recalling some facts about the $\tau$-Wigner peudo-differential calculus, see [31]

For $\tau \in[0,1]$ define the torsion operator $T_{\tau}: \Phi(x, y) \longrightarrow \Phi(x+\tau y, x-(1-\tau) y)$. The $\tau$-Wigner transform of $f, g \in L^{2}\left(\mathbb{R}^{d}\right)$ is

$$
W i g_{\tau}(f, g)(x, \omega)=\int_{\mathbb{R}^{d}} e^{-2 \pi i t \cdot \omega} f(x+\tau t) \overline{g(x-(1-\tau) t)} d t=\mathcal{F}_{2}\left[T_{\tau}(f \otimes \bar{g})\right]
$$

whereas the $\tau$-Weyl operator with symbol $b_{\tau}(x, \omega)$ is

$$
W_{\tau}^{b_{\tau}}: f \longrightarrow W_{\tau}^{b_{\tau}} f(x)=\int_{\mathbb{R}^{2 d}} e^{-2 \pi i(x-y) \cdot \xi} b_{\tau}((1-\tau) x+\tau y, \xi) f(y) d y d \xi .
$$

In our result will be crucial the fact that the map $b_{\tau} \longrightarrow W_{\tau}^{b_{\tau}}$ is injective.

The $\tau$-Wigner transforms and $\tau$-Weyl operators are related by the formula

$$
\left(W_{\tau}^{b_{\tau}} f, g\right)=\left(b_{\tau}, W_{i} g_{\tau}(g, f)\right)
$$

The connection between the $\tau$-symbol $b_{\tau}$ of the operator $W_{\tau}^{b_{\tau}}$ and its Schwartz kernel $K_{\tau}$ is given by:

$$
\begin{aligned}
& K_{\tau}(x, y)=\mathcal{F}_{\xi \rightarrow x-y}\left[b_{\tau}((1-\tau) x+\tau y, \xi)\right] \\
& b_{\tau}(v, \xi)=\mathcal{F}_{w \rightarrow \xi}\left[K_{\tau}(v+\tau w, v-(1-\tau) w)\right]=\mathcal{F}_{2}\left[T_{\tau}\left(K_{\tau}\right)\right]
\end{aligned}
$$

We remark that when $K_{\tau}=f \otimes \bar{g}$, we have

$$
b_{\tau}=\operatorname{Wig}_{\tau}(f, g) .
$$

Let $a, b \in \mathbb{R}_{+}^{d}$, for a function $f(x, \xi)$ defined on $\mathbb{R}^{2 d}$ we define the periodization

$$
P_{a, b} f(x, \xi)=\sum_{h, k \in \mathbb{Z}} T_{(a h, b k)} f(x, \xi)=\sum_{h, k \in \mathbb{Z}} f(x-a h, \xi-b k) .
$$


As proved in section 0.6, given two window functions $g, \gamma$, the Gabor operator formally defined as

$$
S_{g, \gamma}: \phi(x) \longrightarrow S_{g, \gamma} \phi(x)=\sum_{h, k}\left(\phi, g_{h, k}\right) \gamma_{h, k}(x),
$$

has Schwartz kernel

$$
K(x, y)=\sum_{h, k} \gamma_{h, k}(x) \overline{g_{h, k}(y)}
$$

where the sum converges in $\mathcal{S}^{\prime}\left(\mathbb{R}^{2 d}\right)$.

\section{Lemma 5.1.}

a) If $\gamma, g \in L_{\varepsilon}^{\infty}\left(\mathbb{R}^{d}\right) \cap \hat{L}_{\varepsilon}^{\infty}\left(\mathbb{R}^{d}\right)$, with $\varepsilon>d / 2$, then $\gamma, g \in M^{1}$.

b) If $\gamma, g \in M^{1}$, then $P_{a, b} W i g_{\tau}(\gamma, g) \in L^{\infty}\left(\mathbb{R}^{2 d}\right)$, for any $a, b>0$.

Proof. a) For $\varepsilon^{\prime}>0$ consider the spaces $L_{\varepsilon^{\prime}}^{2}\left(\mathbb{R}^{d}\right)$ which consists of the measurable functions $f$ on $\mathbb{R}^{d}$ for which the norm $\|f\|_{L_{\varepsilon^{\prime}}^{2}}=\left(\int_{\mathbb{R}^{d}}|f(x)|^{2}\langle x\rangle^{2\left(d+\varepsilon^{\prime}\right)} d x\right)^{1 / 2}$ is finite. Choosing $0<\varepsilon^{\prime}<\varepsilon-d / 2$, by an easy calculation we obtain $\|\cdot\|_{L_{\varepsilon^{\prime}}^{2}} \leq$ $\|\cdot\|_{L_{\varepsilon}^{\infty}}\left(\int\langle x\rangle^{2\left(\varepsilon^{\prime}-\varepsilon\right)} d x\right)^{1 / 2}$ and therefore $L_{\varepsilon}^{\infty}\left(\mathbb{R}^{d}\right) \hookrightarrow L_{\varepsilon^{\prime}}^{2}\left(\mathbb{R}^{d}\right)$. The conclusion follows now from the well-known fact that $L_{\varepsilon^{\prime}}^{2}\left(\mathbb{R}^{d}\right) \cap \hat{L}_{\varepsilon^{\prime}}^{2}\left(\mathbb{R}^{d}\right) \hookrightarrow M^{1}$ (see e.g. [15], Prop. 12.1.6).

b) If $\gamma, g \in M^{1}$, then $W i g_{\tau}(\gamma, g) \in W\left(\mathbb{R}^{2 d}\right)$, ( [15], Prop. 12.1.11), (which is actually a characterization of $\left.M^{1}\right)$, and this in turn implies that the periodization $P_{a, b} W_{i g}(\gamma, g)$ is in $L^{\infty}\left(\mathbb{R}^{2 d}\right)([15]$, Lemma 6.1.2).

Proposition 5.2. Consider $\gamma, g \in M^{1}$, then the $\tau$-symbol of the operator $S_{g, \gamma}$ belong to $L^{\infty}\left(\mathbb{R}^{d}\right)$ and has the following expression

$$
b_{\tau}(x, \xi)=P_{a, b} W i g_{\tau}(\gamma, g)(x, \xi) .
$$

Proof. We notice at first that, from Lemma $5.1 \mathrm{~b})$, the periodization $P_{a, b} W i g_{\tau}(\gamma, g)$ is in $L^{\infty}\left(\mathbb{R}^{2 d}\right)$. We show now the covariance property of the $\tau-$ Wigner transform. For $g, \gamma \in L^{2}\left(\mathbb{R}^{d}\right)$, we have

$$
\begin{aligned}
W i g_{\tau}\left(\gamma_{h, k}, g_{h, k}\right)(x, \xi) & =W i g_{\tau}\left(M_{k} T_{h} \gamma, M_{k} T_{h} g\right)(x, \xi) \\
& \left.=W i g_{\tau}\left(T_{h} \gamma, T_{h} g\right)(x, \xi-\tau b k-(1-\tau) b k)\right) \\
& =W i g_{\tau}(\gamma, g)(x-(1-\tau) a h-\tau a h, \xi-b k) \\
& =W i g_{\tau}(\gamma, g)(x-a h, \xi-b k) .
\end{aligned}
$$

Therefore $W i g_{\tau}\left(\gamma_{h, k}, g_{h, k}\right)(x, \xi)=W i g_{\tau}(\gamma, g)(x-a h, \xi-b k)$ for every $\tau \in[0,1]$.

By (5.3) the operator $S_{\gamma, g}$ has Schwartz kernel $K(x, y)=\sum_{h, k} \gamma_{h, k}(x) \overline{g_{h, k}(y)}$ and by (5.2) its $\tau$-symbol is

$$
\begin{aligned}
b_{\tau}(x, \xi) & =\mathcal{F}_{\in}\left[T_{\tau} \sum_{h, k} \gamma_{h, k}(x) \overline{g_{h, k}(y)}\right] \\
& =\sum_{h, k} \mathcal{F}_{\in}\left[T_{\tau}\left(\gamma_{h, k}(x) \overline{g_{h, k}(y)}\right)\right] \\
& =\sum_{h, k} W_{i g_{\tau}}\left(\gamma_{h, k}, g_{h, k}\right)(x, \xi) \\
& =\sum_{h, k} W_{i g_{\tau}}(\gamma, g)(x-x h, \xi-b \xi) \\
& =P_{a, b} W_{i}(\gamma, g)(x, \xi) .
\end{aligned}
$$

Proposition 5.3. Let $\gamma \in M^{1}$ and suppose that $\left\{\gamma_{h, k}\right\}$ is a Gabor frame in $L^{2}\left(\mathbb{R}^{d}\right)$, then for a Gabor system $\left\{g_{h, k}\right\}$ with $g \in M^{1}$ the following are equivalent:

(a) $\left\{g_{h, k}\right\}$ is a dual frame of $\left\{\gamma_{h, k}\right\}$,

(b) $P_{a, b} W i g_{\tau}(\gamma, g) \equiv 1$ idendically on $\mathbb{R}^{2 d}$. 
Proof. $\left\{g_{h, k}\right\}$ is a dual frame of $\left\{\gamma_{h, k}\right\}$ if and only if the operator $S_{g, \gamma}=I d$. From the injectivity of the correspondence between $\tau$-symbols and operators and by Proposition 5.2, this happens if and only if $P_{a, b} W i g_{\tau}(\gamma, g) \equiv 1$ idendically on $\mathbb{R}^{2 d}$.

The property above can be reformulated using more general representations in the Cohen class, which is the set of time-frequency representations defined as quadratic forms of the type

$$
f(x) \longrightarrow Q_{\sigma}(f)(x, \xi)=(\sigma * W i g(f))(x, \omega) .
$$

Here $\sigma(x, \xi)$ is the so-colled Cohen kernel (note that Wig $(f)$ is obtained for $\sigma=\delta$, the Dirac delta), see e.g. [3], [5], [15].

Clearly $f(x)$ and $\sigma(x, \xi)$ must be chosen in functional (or distributional) spaces such that the convolution in the time-frequency space $\mathbb{R}_{x}^{d} \times \mathbb{R}_{\xi}^{d}$ appearing in (5.4) makes sense.

Proposition 5.4. In the same hypothesis of Proposition 5.3, suppose $\sigma \in \mathcal{E}^{\prime}\left(\mathbb{R}^{2 d}\right) \cup$ $L^{1}\left(\mathbb{R}^{2 d}\right)$ and $\mathcal{F}^{-1}(1 / \widehat{\sigma}) \in \mathcal{E}^{\prime}\left(\mathbb{R}^{2 d}\right) \cup L^{1}\left(\mathbb{R}^{2 d}\right)$, where $\mathcal{E}^{\prime}\left(\mathbb{R}^{2 d}\right)$ is the space of compactly supported distributions on $\mathbb{R}^{2 d}$. Then the following are equivalent:

(a) $\left\{g_{h, k}\right\}$ is a dual frame of $\left\{\gamma_{h, k}\right\}$ modulo a multiplicative constant,

(b) $P_{a, b} Q_{\sigma}(\gamma, g)$ is constant on $\mathbb{R}^{2 d}$.

Proof. Let $g_{h, k}$ be multiple of a dual frame of $\gamma_{h, k}$, then by Proposition 5.3 with $\tau=1 / 2$ we have that $P_{a, b} W i g(\gamma, g)$ is constant. As $Q_{\sigma}=\sigma * W i g(\gamma, g)$ we have

$$
\begin{aligned}
P_{a, b} Q_{\sigma}(\gamma, g) & =\sum_{h, k} T_{(a h, b k)}(\sigma * W i g(\gamma, g)) \\
& =\sum_{h, k} \sigma *\left(T_{(a h, b k)} \operatorname{Wig}(\gamma, g)\right) \\
& =\sigma * \sum_{h, k} T_{(a h, b k)} \operatorname{Wig}(\gamma, g) \\
& =\sigma * P_{a, b} \operatorname{Wig}(\gamma, g) \\
& =c
\end{aligned}
$$

with $c=\int_{\mathbb{R}^{2 d}} \sigma(x, \omega) d x d \omega$. We remark that the convolutions above make sense either considering $\sigma \in \mathcal{E}^{\prime}\left(\mathbb{R}^{2 d}\right)$ and the constant $P_{a, b} W i g(\gamma, g) \in \mathcal{E}\left(\mathbb{R}^{2 d}\right)$ or $\sigma \in$ $L^{1}\left(\mathbb{R}^{2 d}\right)$ and $P_{a, b} W i g(\gamma, g) \in L^{\infty}\left(\mathbb{R}^{2 d}\right)$.

Viceversa suppose that (b) holds and consider the Weyl symbol $b_{1 / 2}(x, \xi)=$ $P_{a, b} W i g(\gamma, g)(x, \xi)$ of the Gabor operator. We have from (5.5)

$$
c=\sigma * b_{1 / 2}
$$

i.e. $b_{1 / 2}=\mathcal{F}^{-1}\left(\frac{c \delta}{\widehat{\sigma}}\right)=c \mathcal{F}^{-1}(\delta) * \mathcal{F}^{-1}(1 / \widehat{\sigma})=c * \mathcal{F}^{-1}(1 / \widehat{\sigma})=c^{\prime}$, which makes sense as $\mathcal{F}^{-1}(1 / \widehat{\sigma}) \in \mathcal{E}^{\prime}\left(\mathbb{R}^{2 d}\right) \cup L^{1}\left(\mathbb{R}^{2 d}\right)$. This means that the Gabor operator is a (multiple) of the identity and therefore (a) holds.

A simple example of Cohen kernel satisfying the hypothesis of the previous proposition is the function $\sigma(x, \xi)=e^{-\alpha|x|-\beta|\xi|}$ with $\alpha, \beta>0$. Actually

$$
\widehat{\sigma}(\omega, y)=\frac{4 \alpha \beta}{\left(\alpha^{2}+4 \pi^{2} \omega^{2}\right)\left(\beta^{2}+4 \pi^{2} y^{2}\right)}
$$

and

$$
\begin{aligned}
\mathcal{F}^{-1}(1 / \widehat{\sigma})(x, \xi) & =\mathcal{F}^{-1}\left(\left(\frac{\alpha}{2}+\frac{4 \pi^{2} \omega^{2}}{2 a}\right)\left(\frac{\beta}{2}+\frac{2 \pi^{2} y^{2}}{a}\right)\right)(x, \xi) \\
& =\left(\frac{\alpha}{2} \delta_{x}-\frac{1}{2 \alpha} \delta_{x}^{\prime \prime}\right)\left(\frac{\beta}{2} \delta_{\xi}-\frac{1}{2 \beta} \delta_{\xi}^{\prime \prime}\right) .
\end{aligned}
$$

Therefore $\mathcal{F}^{-1}(1 / \widehat{\sigma})$ belongs to $\mathcal{E}^{\prime}\left(\mathbb{R}^{2}\right)$ and we have

$$
c * \mathcal{F}^{-1}(1 / \widehat{\sigma})=\text { constant. }
$$




\section{REFERENCES}

[1] Á. Bényi, K. Gröchenig, C. Heil, and K. Okoudjou, Modulation spaces and a class of bounded multilinear pseudodifferential operators, J. Operator Theory, 54 (2005), 387-399.

[2] H. Bölcskei, A.J.E.M. Janssen, Gabor frames, unimodularity, and window decay, J. Fourier Anal. Appl., 6 n.3, (2000), 255-276.

[3] P. Boggiatto, G. De Donno and A. Oliaro, Time-Frequency representations of Wigner type and pseudo-differential operators, Trans. Amer. Math. Soc. 362 n.9,(2010), 4955-4981.

[4] P. Casazza, O. Christensen, A. J. E. M. Janssen, Weyl-Heisenberg Frames, translation invariant systems and the Walnut representation, J. Funct. An., 180 n.1, (2001), 85-147.

[5] L. Cohen, Time-Frequency Analysis, Prentice Hall Signal Proc. series, New Jersey, 1995.

[6] E. Cordero, M. de Gosson., F. Nicola, Time-frequency analysis of Born-Jordan pseudodifferential operators, J. Funct. An., 272 (2017), 577-598.

[7] O. Christensen, H.O. Kim, R.Y. Kim, Gabor windows supported on [-1,1] and compactly supported dual windows, Appl. Comput. Harmon. Anal. 28, (2010), 89-103.

[8] O. Christensen, An Introduction to Frames and Riesz Bases, Birkäuser, Boston MA 2016.

[9] O. Christensen, B. Deng, C. Heil, Density of Gabor frames, Appl. Comput. Harmon. Anal. 7, (1999), 292-304.

[10] H. G. Feichtinger, Gabor expansions of signals: computational aspects and open questions. Landscape of Time-Frequency Analysis, ATFA Conference 2017, Springer, to appear.

[11] H. G. Feichtinger, W. Kozek, Quantization of lattice-invariant operators on elementary LCA groups. In Gabor Analysis and Algorithms, Appl. Numer. Harmon. Anal., Birkäuser, Boston MA 1998, 233-266.

[12] H. G. Feichtinger, G. Zimmermann, A Banach space of test functions for Gabor analysis. In Gabor Analysis and Algorithms, Appl. Numer. Harmon. Anal., Birkäuser, Boston MA 1998, 123-170.

[13] G. Folland, A Course in Abstract Harmonic Analysis, CRC Press, 1995.

[14] S.R. Ghoparde, B.V. Limaye, A Course in Multivariate Calculus and Analysis, Undegraduate texts in math. Springer, 2010.

[15] K. Gröchenig, Foundations of Time-Frequency Analysis, Birkäuser, Boston 2001.

[16] K. Gröchenig, The mystery of Gabor frames, J. Fourier Anal. Appl., 20 n. 4 (2014), 865-895.

[17] K. Gröchenig, J. L. Romero, J. Stöckler, Sampling theorems for shift-invariant spaces, Gabor frames, and totally positive functions, Invent. Math., 211 n.3, (2018), 1119-1148.

[18] K. Gröchenig, J. Stöckler, Gabor frames and totally positive functions, Duke Math. J., 162 n.6, (2013), 1003-1031.

[19] C. Heil, History and evolution of the density theorem for Gabor frames, J. Fourier Anal. Appl., 13 n. 2 (2007), 113-166.

[20] C. Heil, A Basis Theory Primer, Birkhäuser, Boston, 2011.

[21] C. Heil, K. Gröchenig, Modulation spaces and pseudodifferential operators, Integral Equations Operator Theory, 34 (1999), 439-457.

[22] I. L. Hwang, The $L^{2}$ boundedness of pseudodifferential operators, Trans. Amer. Math. Soc. 302 n.1(1987), 55-76.

[23] A. J. E. M. Janssen, On generating tight Gabor frames at critical density, J. Fourier Anal. Appl., 9 n. 2 (2003), 175-214.

[24] A. J. E. M. Janssen, Zak transforms with few zeros and the tie, Advances in Gabor Analysis, Appl. Num. Harm. Anal., Birkhäuser, Boston, MA, (2003), 31-70.

[25] A.J.E.M. Janssen, T. Strohmer, Hyperbolic secants yield Gabor frames, Appl. Comput. Harmon. Anal., 12, n.3, (2002), 259-267.

[26] D. Labate, Pseudodifferential operators on modulation spaces, J. Math An. Appl., 262 n.1 (2001), 242-255.

[27] H. Landau, On the density of phase space expansions, IEEE Trans. Inform. Theory, 39, (1993), 1152-1156.

[28] Y.I. Lyubarskii, Frames in the Bargman space of entire functions, in Entire and subharmon. funct., Am. Math. Soc., Providence, RI, (1992), 167-180.

[29] A. Ron, Z. Shen, Weyl-Heisenberg frames and Riesz bases in $L^{2}\left(\mathbb{R}^{d}\right)$, Duke Math. J., 89 n.2 (1997), 237-282.

[30] K. Seip, R. Wallsten, Density theorems for sampling and interpolation in the Bargman-Fock space, II, J. Reine Angew. Math., 429, (1992), 107-113.

[31] M.A. Shubin, Pseudodifferential Operators and Spectral Theory, Springer-Verlag, Berlin, 1987.

[32] J. Toft, Continuity properties for modulation spaces with applications to pseudo-differential calculus i. , J. Funct. Anal., 207 (2), (2004), 399-429. 
[33] J. Toft, Continuity properties for modulation spaces with applications to pseudo-differential calculus ii., Ann. Global Anal. Geom., 26, (2004), 73-106.

[34] L. Rodino, P. Wahlberg, The Gabor wave front set, Monatshefte für Mathematik (Print), 173, (2014), 625-655.

[35] D. F. Walnut, Continuity properties of the Gabor frame operator, J. Math. Anal. Appl., 165 n.2, (1992), 479-504.

Dipartimento di Matematica Università di Torino, Via Carlo Alberto 10, I-10123, TORIno,, ITALY

E-mail address: paolo.boggiatto@unito.it

Dipartimento di Matematica, Università di Torino, Carlo Alberto 10, I-10123, TORINo, Italy

E-mail address: gianluca.garello@unito.it 